\title{
Experimental Characterization of LTE Wireless Links in High-Speed Trains
}

\author{
Tomás Domínguez-Bolaño, José Rodríguez-Piñeiro, José A. García-Naya, and Luis Castedo
}

Universidade da Coruña, A Coruña, Spain

Correspondence should be addressed to Tomás Domínguez-Bolaño; tomas.bolano@udc.es

Received 25 May 2017; Accepted 3 August 2017; Published 28 September 2017

Academic Editor: Yin Xuefeng

Copyright (C) 2017 Tomás Domínguez-Bolaño et al. This is an open access article distributed under the Creative Commons Attribution License, which permits unrestricted use, distribution, and reproduction in any medium, provided the original work is properly cited.

\begin{abstract}
Multimedia and data-based services experienced a nonstopping growth over the last few years. People are continuously on the move using devices to access multimedia contents or other data-based services. Due to this, railway companies are showing a great interest in deploying broadband mobile wireless networks in high-speed-trains with the aim of supporting both passenger services provisioning as well as automatic train control and signaling. Nowadays, the most widely used technology for communications between trains and the railway infrastructure is GSM for Railways (GSM-R); however, it has limited capabilities to support such advanced services. Due to its success in the mass market, Long Term Evolution (LTE) seems to be the best candidate to substitute GSM-R. In this paper, we experimentally characterize the downlink between an LTE Evolved NodeB (eNodeB) and a high-speed train in a commercial high-speed line. We consider two links: the one between the eNodeB and the antennas placed outdoors on the train roof, and the direct link between the eNodeB and a receiver inside the train. Such a characterization consists in assessing the path loss, the Signal to Noise Ratio, the K-Factor, the Power Delay Profile, the delay spread, and the Doppler Power Spectral Density.
\end{abstract}

\section{Introduction}

Railway communications can be divided into two groups: (a) train control signaling and safety-related communications and (b) noncritical communications, both for train staff and passengers. While the first kind of communications usually does not require high data rates, it in turn imposes stringent constraints to the Quality of Service (QoS) [1-5]. Operational and functional requirements for the railway environment require in fact reduced delays or call setup times, as well as very reduced service interruptions. The second type of railway communications demands higher throughputs (e.g., access to multimedia services), but they do not impose so strict requirements in terms of reliability.

Nowadays, the most widely used technology for train control signaling and safety-related communications between trains is based on GSM for Railways (GSM-R) and requires a specific network deployment with base stations located along the track. The GSM-R, which is based on the mature Global System for Mobile Communications (GSM) technology, provides a continuous communication channel between the train driver and the ground controllers, being well-suited to perform emergency calls, selective phone calls, or small data transmission. However, GSM-R has reduced capabilities to support more advanced services, such as automatic pilot or provisioning broadband communications to train staff.

Besides that, multimedia and data-based services experienced a nonstopping growth over the last few years [1]. People are continuously on the move using devices to access multimedia contents or other data-based services. Whereas sometimes the transmission of a few data bits is enough, continuous streaming of multimedia data is required in other situations. In most cases, users are connected to generalpurpose mobile networks. However, railway companies are aware of the market opportunity and aim to provide passengers with multimedia services and/or broadband Internet access onboard. The Mobile-Relay technique has been proposed in [6] as a way to provide coverage to passengers based on a relaying scheme (a link from the cellular base station to 
an external antenna installed on the train, which is relayed to a local base station inside the train carriage).

Taking into account the big success of Long Term Evolution (LTE) in the evolution of mobile networks, it seems reasonable to consider LTE as the best candidate to substitute GSM-R as the fundamental technology for railway communications, as well as provisioning services to passengers. While in previous publications we studied the suitability of LTE to fulfill the operational and functional requirements for the railway environment (e.g., see [1-4]), in this paper, we account for the detailed characterization of the wireless link based on LTE measurements in a high-speed train line in Spain. We consider both the channel between an Evolved NodeB (eNodeB) and a passenger inside a highspeed train, as well as the direct channel between the eNodeB and the outdoor antennas installed on the train carriage.

The first step for migrating GSM-R into LTE is to assess the performance of such a communications system for the railway environment. Knowledge of the wireless channel characteristics is the fundamental basis for the planning of wireless communication networks and the design of transceivers [1, 7]. However, HST communications demand for the evaluation of specific scenarios which are typical in most railway lines, such as [1] rural macrocell, hilly terrain, viaducts, cuttings, or tunnels. Hence, in recent years, a lot of attention has been put to measurement-based analysis to characterize the different types of high-speed railway scenarios. In [8-11], several parameters of the channel were analyzed and modeled for a viaduct scenario based on measurements carried out using GSM-R Base Stations (BSs) at $930 \mathrm{MHz}$. In [12], the channel is characterized and modeled for a cutting scenario based on measurements carried out using GSM-R BSs at $930 \mathrm{MHz}$. The papers [13-15] analyze the channel for a viaduct scenario considering a carrier frequency of $2.35 \mathrm{GHz}$. In [16], a hilly terrain scenario is considered and the delay and Doppler spread is characterized for a carrier frequency of 2.4 GHz. In [17], the authors use the commercially deployed LTE base stations along the HST railway between Beijing and Shanghai (with a total distance of $1318 \mathrm{~km}$ ) to characterize statistically the parameters of the observed channels.

In our case, we did not perform the measurements by employing a general-purpose mobile network deployment during the train operation. However, different from other works, we installed an eNodeB taking advantage of the available infrastructure for GSM-R in the considered highspeed train line. We considered a carrier frequency of $2.6 \mathrm{GHz}$ and the $10 \mathrm{MHz}$ profile of LTE. Furthermore, we deployed two sectors, and the corresponding eNodeB antenna panels were placed in the same tower where the GSM-R antennas are installed. Since we measured LTE standard-compliant signals, tasks like time and frequency synchronization or channel estimation were performed considering exclusively the same information that a commercial receiver would use (i.e., synchronization signals and pilots). We could also perform different train passes along a segment of about $7 \mathrm{~km}$ long centered at the $\mathrm{eNodeB}$ site with distinct train speeds and varying the data traffic. At the receiver (train) side, we could access the external antennas of the train, being able to evaluate the eNodeB-train wireless link in a realistic way. We also considered antennas installed indoors to model the direct eNodeB-passenger link. Furthermore, high-resolution results are obtained from our measurements (which are freely available for other research groups), since we continuously capture the signal at the receiver during the whole measurement campaigns.

The main contribution of this paper is the complete and detailed characterization of the downlink between a commercial LTE eNodeB and a train moving at high velocities along a track in commercial operation in a rural area in Spain. We consider two links: the one between the eNodeB and the antennas placed outdoors on the train roof and the direct link between the eNodeB and a receiver inside the train. Such a characterization consists in assessing the path loss, the Signal to Noise Ratio (SNR), the K-Factor, the Power Delay Profile (PDP), the delay spread, and the Doppler Power Spectral Density (PSD) for different train speeds. Finally, we showed the specific characteristics of the railway channel and the impact of the train speed on them. Besides that, the mathematical description of all the procedures followed to obtain the results is detailed. In particular, mathematical descriptions in both discrete time and frequency are provided for all the estimated parameters: channel response, synchronization, SNR, path loss, $K$-Factor, PDP, delay spread, and Doppler PSD. Additionally, guidelines about the drawbacks and pitfalls to be considered for the different analysis methods are provided.

The structure of the paper is as follows: Section 2 describes in detail the environment, as well as the experimental setup and procedure considered for the evaluations. Section 3 explains the basic aspects of the signal processing performed at the receiver, including the signal synchronization, or the channel and SNR estimation. Section 4 characterizes the path loss for both the eNodeB-train and direct eNodeB-passenger links, while Section 5 studies the eNodeB-train channel condensed parameters, such as the $K$ Factor, the PDP, the delay spread, and the Doppler PSD. In Section 6, we study significant regions of the train path in detail, showing how train infrastructure elements can impact on the wireless link. Finally, Section 7 concludes the paper.

\section{Experimental Setup}

We evaluated experimentally the links between an eNodeB and receive antennas placed on the roof of a train carriage, as well as a mobile receiver inside the train. By first evaluating the path loss, we motivate the need of a relay architecture to distribute the signal inside the train through repeaters or Access Points (Aps), while employing external (trainmounted) antennas for the link between the train and the eNodeB. Then, the effects of the high-speed on the latter link are studied in detail by means of parameters such as the $K$ Factor, the PDP, or the Doppler PSD, taking into account the effects at high speeds.

A measurement campaign was conducted in a high-speed train line in commercial operation. Different from other works, we could access the railway environment and equipment, which enabled us to perform different experiments during maintenance periods (mainly overnight) by freely 

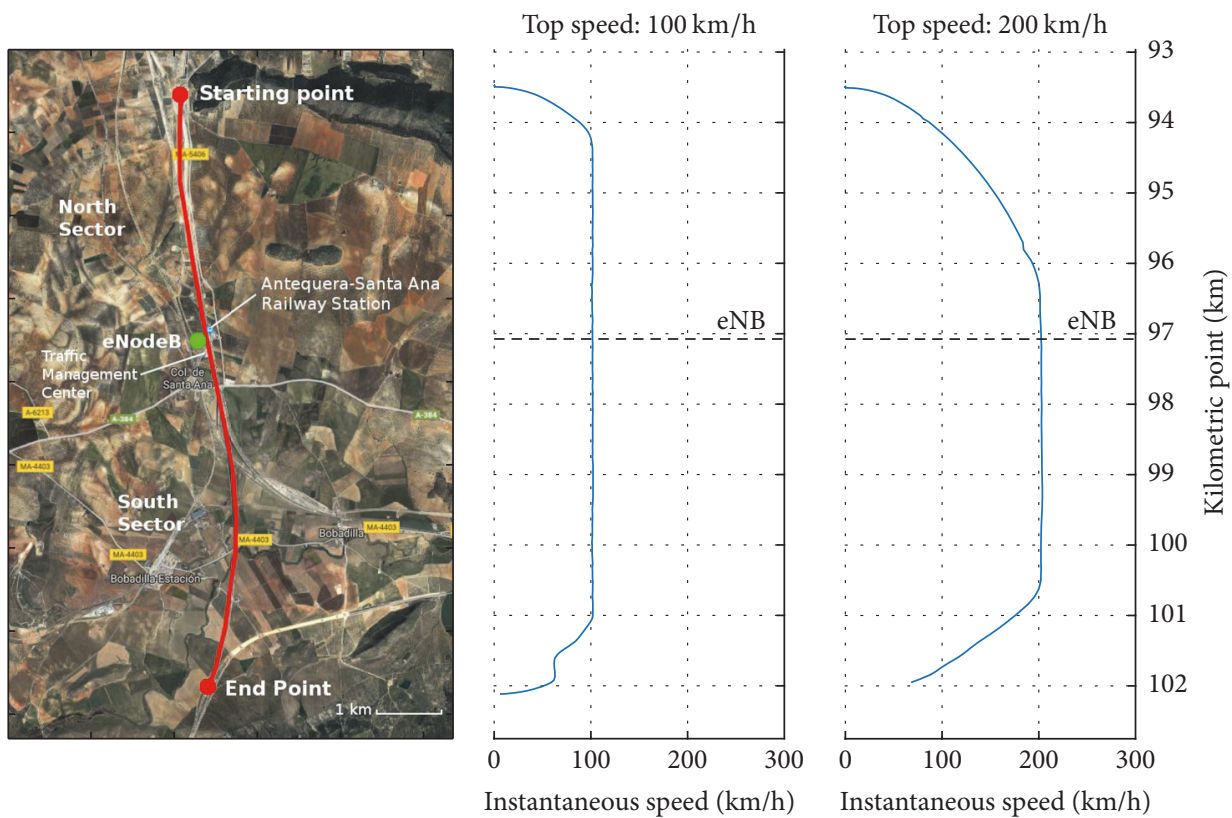

FiguRE 1: Map of the scenario considered for the measurements. The instantaneous train speed is shown for each point of the trajectory, both for the cases considering a maximum speed of $100 \mathrm{~km} / \mathrm{h}$ and $200 \mathrm{~km} / \mathrm{h}$. The position of the eNodeB is also specified. Map Image by @2017 DigitalGlobe, Cartography Institute of Andalucía; Map Data by @2017 Google, National Geographic Institute of Spain.

controlling the train movement as well as the transmitted signals. Even more, one of the most important features of our experiments is that, instead of using eNodeB deployed for general-purpose mobile cellular communications, we installed an eNodeB in one of the GSM-R sites specifically devoted for railway communications. Furthermore, we obtained the permission to employ the carrier frequency at $2.6 \mathrm{GHz}$, which is commercially used in such an area. Measurements were carried out along a track segment of approximately $7 \mathrm{~km}$ long centered at the GSM-R site in a rural area. The considered train was specifically designed to test all the systems each time a new line is opened; hence, the access to some resources such as the external antennas installed on its roof was granted. This allowed us to analyze the use of LTE for high-speed train applications at the actual environment and employing the specific infrastructure of the railway operator. It is also worth noting that the transmit power was high enough so that the SNR level exceeds $20 \mathrm{~dB}$ (when using antennas placed outdoors). This allows for correctly synchronizing most of the LTE frames without errors, hence enabling us to analyze the signal along the whole train path: that is, instead of only sampling the acquired signal during short time intervals, while the train moves along the track, our equipment acquires the transmitted LTE frames in a continuous fashion. The following subsections describe the details of the measurement environment, the equipment used, and the methodology followed to perform the measurements.

\subsection{Measurement Environment}

2.1.1. Test Track. The considered high-speed train line connects Córdoba and Málaga (Spain) and is designed to cope with speeds up to $330 \mathrm{~km} / \mathrm{h}$. The measured segment is located between the Kilometric Points (KPs) 93.0 and 102.0, whereas the GSM-R site is located at KP 97.075 (the exact Global Positioning System (GPS) coordinates of the site are $37^{\circ} 4^{\prime} 3.14^{\prime \prime} \mathrm{N}, 4^{\circ} 43^{\prime} 12.52^{\prime \prime} \mathrm{W}$ ), as imaged in Figure 1. The Antequera-Santa Ana Railway Station (KP 96.800) and a Traffic Management Center are also located in the vicinity of the GSM-R site.

2.1.2. Test Train. The considered test train was the so-called Séneca laboratory train (Talgo A-330), provided by the Spanish Railway Infrastructure Administrator (ADIF), imaged in Figure 2(a). The train was intended for infrastructure inspections and it was extensively used in testing activities for the deployment of the European Rail Traffic Management System/European Train Control System (ERTMS/ETCS) standard in Spanish high-speed lines. Electrically powered, the train can reach a maximum speed of $363 \mathrm{~km} / \mathrm{h}$, although the maximum considered speed for our measurements was limited to $200 \mathrm{~km} / \mathrm{h}$ because the time (and also the distance) required to reach a higher speed is extremely large, thus limiting the number of trials to be performed in a typical measurement session during the night. The train is $80.92 \mathrm{~m}$ long and includes an inner panel (see Figure 2(b)) which allows for the connection to the external antennas on its roof (see Figure 2(c)): two of them are multiband antennas (800, 900, 1800, 1900, UMTS, UMTSII, W-LAN, and GPS) Kathrein 870 10003, whereas the other two are Kathrein 87010007 (without GPS), both featuring a $0 \mathrm{~dB}$ gain and a Voltage Standing Wave Ratio (VSWR) smaller than 2.0:1 at the considered carrier frequency of $2.6 \mathrm{GHz}$. During the measurements we used two external antennas, one of each type. The GPS connection is used by the GPS-disciplined 


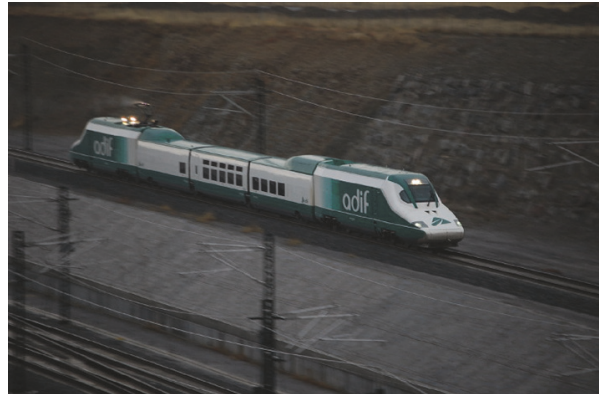

(a) Photography of the whole train

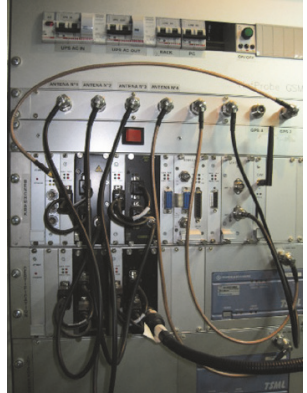

(b) Inner panel for connection to the outdoor antennas

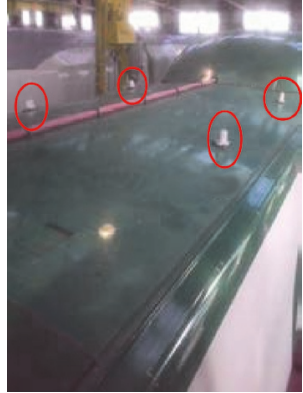

(c) Outdoor antennas on the roof of the train carriage (Kathrein 87010003 and 870 10007). The four antennas are marked with red ellipses

Figure 2: Séneca laboratory train (Talgo A-330).

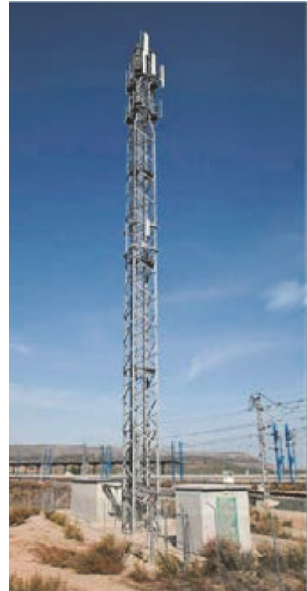

(a) Image of the tower and the two cabins for telecom munications equipment

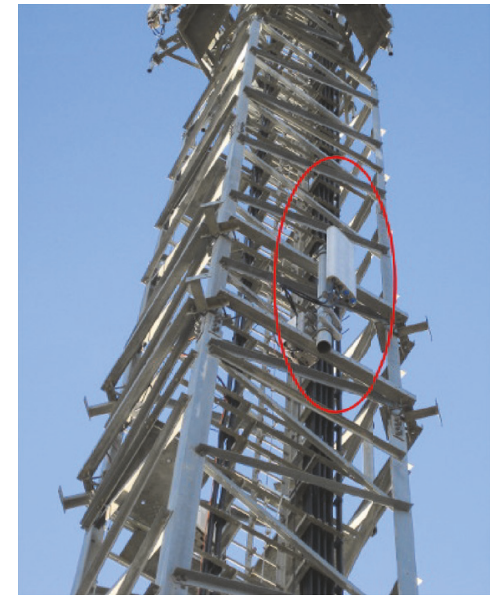

(b) Antenna installed at the tower (for south sector)

FIGURE 3: Pictures of the GSM-R site.

oscillators included in the measurement equipment and to match the acquired samples with the estimated position and velocity of the train.

\subsection{Measurement Equipment}

2.2.1. Ground Equipment. The GSM-R site, imaged in Figure 3(a), includes a $40 \mathrm{~m}$ height tower with antennas for different wireless technologies and two cabins where the communications equipment is installed. A commercial LTE eNodeB was set up inside one of the available cabins and two remote radio heads were mounted at the tower structure, each one connected to two antenna elements of a different antenna panel (see Figure 3(b)). Such antenna panels (Moyano MYDTBSBS17276518) were placed at a height of $20 \mathrm{~m}$ and orientated as the available GSM-R antennas. At the considered carrier frequency $(2.6 \mathrm{GHz})$, these cross-polarized antennas
TABLE 1: Ground antennas orientation, in both vertical (with respect to the horizontal plane) and horizontal (with respect to the north direction) dimensions.

\begin{tabular}{lcc}
\hline Sector & \multicolumn{2}{c}{ Antenna orientation $\left[^{\circ}\right]$} \\
& Azimuth & Elevation \\
\hline North & 355 & 0 \\
South & 175 & -1.4 \\
\hline
\end{tabular}

feature a gain of $18 \mathrm{dBi}$ and a half-power beam width of $62^{\circ}$ (horizontal) and $5^{\circ}$ (vertical), as imaged in the radiation pattern shown in Figure 4. Each antenna panel is used to deploy a sector; hence, two sectors are used, named as "north sector" and "south sector," respectively (see Figure 1). Table 1 shows the orientation of the ground antennas. 


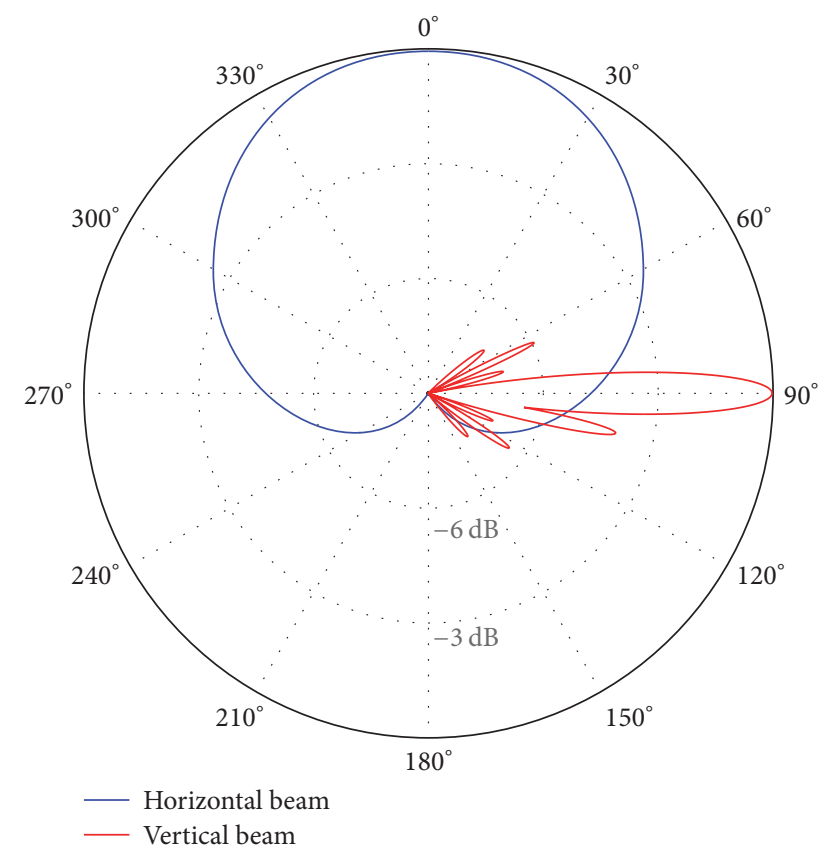

FIGURE 4: Radiation pattern for the transmit antennas (Moyano MY-DTBSBS17276518). Both the vertical and horizontal radiation patterns are shown.

The eNodeB was configured to transmit an LTE signal with the profile of $10 \mathrm{MHz}$ bandwidth, transmit diversity (a single transmit block is encoded and transmitted through two different antennas. Precoding is performed in order to separate the two different antennas) and normal cyclic prefix (CP) length (the first Orthogonal Frequency-Division Multiplexing (OFDM) symbol of an LTE slot (a slot occupies half a subframe) has a cyclic prefix length of $N_{\text {long }}^{\mathrm{CP}}=80$ samples (5.2083 microseconds), whereas for the remaining six OFDM symbols the cyclic prefix is reduced to $N_{\text {short }}^{\mathrm{CP}}=$ 72 samples (4.6875 microseconds)). More details regarding the transmit signal configuration are specified in Table 2. Note that two configurations for data traffic transmission were considered (a commercial LTE receiver (different than those employed to produce the results shown in the paper) is connected as the only user to the eNodeB; this way we can capture the signals between the eNodeB and the commercial receiver to be used for our evaluations):

(i) Maximum throughput: all the resource blocks of the LTE signal are filled in with data. Hence, the maximum payload of LTE is used for the configuration parameters specified in Table 2.

(ii) ETCS + VoIP: a stream of European Train Control System (ETCS) data traffic is transmitted in parallel with a Voice over Internet Protocol (VoIP) call.

In both cases, the receiver used in the measurements has no a priori knowledge about the transmit data.

2.2.2. Onboard Equipment. The onboard equipment consists of two nodes from the so-called GTEC Testbed (described
TABLE 2: LTE testbed configuration parameters.

\begin{tabular}{ll}
\hline Parameter & Value \\
\hline Sampling frequency, $f_{s}$ & $15.36 \mathrm{MHz}$ \\
Transmit power & $46 \mathrm{dBm}$ \\
Maximum antenna gain & $18 \mathrm{dBi}(\mathrm{TX}), 0 \mathrm{dBi}(\mathrm{RX})$ \\
Carrier frequency & $2.6 \mathrm{GHz}$ \\
Bandwidth & $10 \mathrm{MHz}(9 \mathrm{MHz}$ occupied) \\
Transmission scheme & Transmission mode 2 (transmit \\
Cyclic prefix length & diversity) [18] \\
\hline
\end{tabular}

in $[19,20])$ operating in receive-only mode. Each of them includes an Ettus/National Instruments Universal Software Radio Peripheral (USRP) B210 (see Figure 5(b)) board connected to a laptop running the GTEC 5G Simulator [20] (see Figure 5(a)) and the Mathworks LTE Toolbox. Notice that the source code of both the GTEC Testbed and the GTEC 5G Simulator is publicly available under the GPLv3 license at https://bitbucket.org/tomas_bolano/gtec_testbed_public.git [21]. Whereas one of the nodes was connected to two external antennas available on the carriage roof, the other was connected to two omnidirectional antennas (MobileMark PSKN3-24/55S) directly attached to the USRP installed inside the carriage. The outdoor antennas allow us to evaluate the link between the eNodeB and the train, while the indoor ones are used to investigate the direct connection between the mobile receiver of a passenger (or staff) and how the eNodeB would work. Additionally, each node was provided with a GPS-disciplined temperature-controlled oscillator (Ettus/National Instruments GPSDO 783454-01) that is also used for georeferencing (time and position) the measurements. Given that the eNodeB also employs a GPS-disciplined oscillator, the frequency offset between the eNodeB and the GTEC Testbed nodes is minimized. Notice that whereas the USRP connected to the external antennas uses the Kathrein 87010003 GPS antenna, the USRP connected to the indoor antennas uses a separate Trimble 66800-40 GPS antenna which is also placed indoors.

2.3. Measurement Procedure. Different train passes were performed at different speeds, in both north and south directions, considering different transmitter configurations and different data traffic types. GPS-based geolocation of the measurements allowed us to accurately combine the results obtained from different train passes. It is worth noting that the obtained results exhibit a very high repeatability level for different train passes with identical configuration parameters. This enables us to compare consistently the results at different speeds. It is also interesting to mention that we capture the data continuously at the receiver during the whole measurements session. This not only requires a huge amount of available storage (approximately $4 \mathrm{~TB}$ of raw data for two measurement sessions of about 3 hours each) but also challenges the measurement equipment in terms of acquisition speed and stability, demanding extremely powerful hardware to process all the data. In this sense, also 


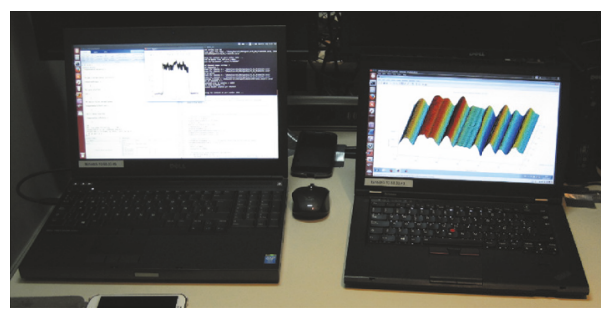

(a) Two laptops mounted onboard running the GTEC 5G Simulator as part of two GTEC Testbed nodes

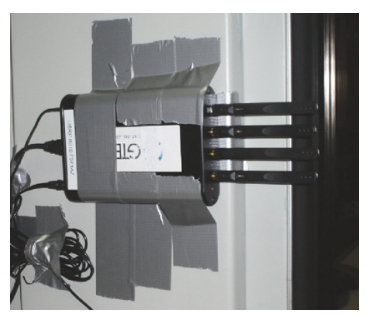

(b) USRP employed as part of a GTEC Testbed node, equipped with the indoor antennas. Only the two antennas in the middle out of the four antennas are used

FIGURE 5: Onboard measurement equipment.

the software developed has to be prepared to cope with such a huge amount of data (details about these aspects can be found in the description of our GTEC 5G Simulator and GTEC Testbed in [20]). The main advantage of our approach is that we can obtain high-resolution results. Additionally, all our measurement results are freely available for other research groups. In order to have access to them please contact us at testbed@gtec.udc.es.

2.3.1. Low Speed Measurements. Train passes at $50 \mathrm{~km} / \mathrm{h}$ were performed to characterize the propagation environment in terms of large-scale parameters. Hence, the SNR and path loss for the antennas placed indoors and outdoors were evaluated. For these measurements, we independently switched on and off the two deployed sectors. This enabled us to measure not only the desired received power in a sector (e.g., received power when the train is in the north sector and only the north sector is active), but also the interference caused to the adjacent sector (e.g., received power when the train is in the north sector and only the south sector is active).

The results presented along this paper were extracted from the samples acquired, while the train speed was kept constant (see Figure 1), whereas an overimposed pattern of gray diagonal lines is used to emphasize the results obtained, while the train was accelerating or decelerating (see Figure 14 as an example).

Figure 6 shows the average SNR per data subcarrier versus the KP. In order to maximize the number of occupied subcarriers devoted to the SNR estimation, the so-called "Maximum Throughput" is considered. The SNR was evaluated as described in Section 3.3 for one antenna placed indoors and another one placed outdoors. It can be seen that obviously the highest SNR values are obtained in the vicinity of the eNodeB. When the train passes in front of the eNodeB, the handover between the two sectors occurs. However, the SNR is higher than $20 \mathrm{~dB}$ for all the points of the train trajectory when the outdoor antennas are used, with the maximum value being about $60 \mathrm{~dB}$. The shape of the SNR curves for the antennas placed indoors is similar, although the received power decreases dramatically due to the penetration losses. On average, a loss of about $26 \mathrm{~dB}$ is measured.

2.3.2. High-Speed Measurements. Once the large-scale parameters were characterized by means of low speed measurements, measurements at higher velocities, such as $100 \mathrm{~km} / \mathrm{h}$

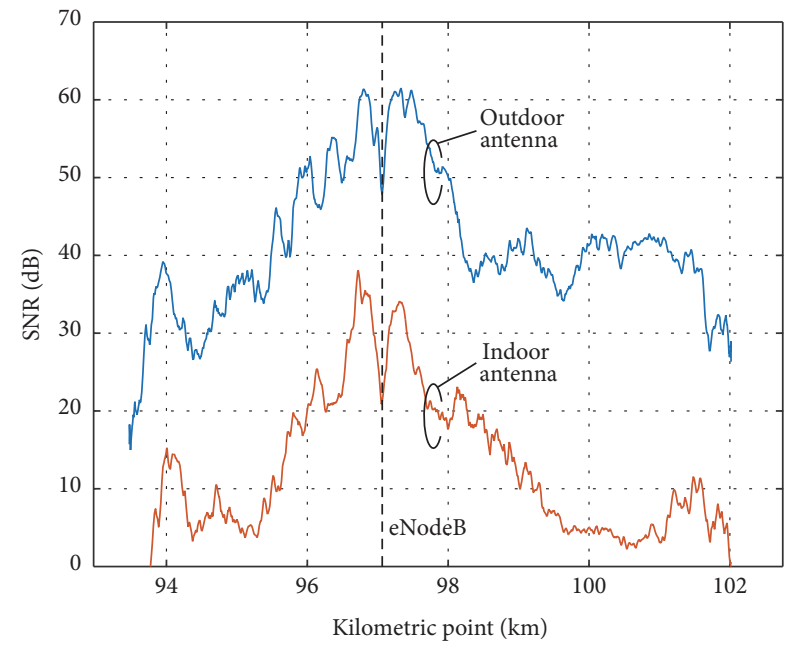

FIGURE 6: Estimated SNR versus KP for one of the indoor and one of the outdoor antennas.

and $200 \mathrm{~km} / \mathrm{h}$, were performed. This way we evaluated the effect of the speed on parameters such as the $K$-Factor, the PDP, and the Doppler PSD. Due to the time required to accelerate and decelerate the train, it was not possible to measure at high speeds during the whole train path. Figure 1 shows the instantaneous speed of the train versus the KP for both measurements cases: $100 \mathrm{~km} / \mathrm{h}$ and $200 \mathrm{~km} / \mathrm{h}$. As shown in Figure 1, the speed is maximum along the central part of the evaluated trajectory. Even for the worst case $(200 \mathrm{~km} / \mathrm{h})$, the evaluations are performed at the maximum speed at least along three kilometers. In this case, the considered model for the data traffic is the one labeled as "ETCS + VoIP" in Section 2.2.1. However, this does not affect the results since only the pilots are used to perform the evaluations.

\section{Signal Processing at the Receiver}

Several relevant parameters are estimated directly from the LTE-compliant acquired signal, such as path loss, PDP, delay spread, and Doppler PSD. Most of the estimates depend on the wireless channel response estimation, which is done by means of the pilot symbols included in the LTE transmit 


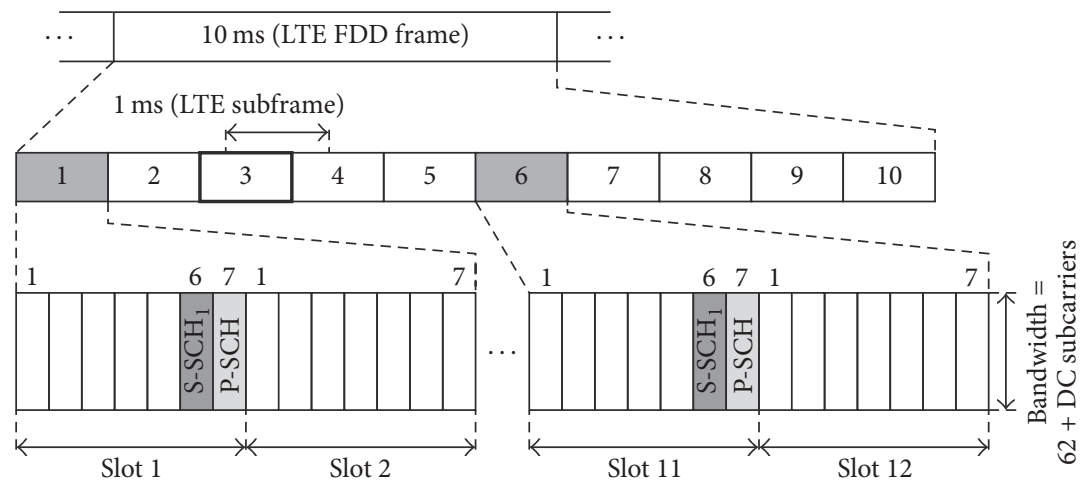

FIGURE 7: Synchronization signals for FDD LTE.

signal. In the following subsections, we detail all the steps followed to process the acquired signals in order to obtain the aforementioned estimates.

3.1. Synchronization in LTE. Before proceeding with the channel response estimation, the first step consists in detecting and synchronizing the LTE signal. LTE provides two synchronization signals, namely, the Primary Synchronization Signal (P-SCH) and the Secondary Synchronization Signal (S-SCH), to enable time and frequency synchronization and to detect the physical cell ID which defines the pilot sequence, as described in [22, Section 6.11]. For FDD LTE signals - the ones considered in our experiments-the P$\mathrm{SCH}$ is transmitted by using the 62 central subcarriers of the last OFDM symbol contained in the first and the eleventh slots of each LTE frame, as shown in Figure 7. This enables the receiver to detect the $\mathrm{P}$-SCH regardless of the configuration of the $\mathrm{CP}$ length. Once the $\mathrm{P}-\mathrm{SCH}$ is detected, the signal is timesynchronized at slot level. While the $\mathrm{P}-\mathrm{SCH}$ is repeated twice per LTE frame, the S-SCH comprises two different signal sequences per each LTE frame, being the first one transmitted in the 62 central subcarriers of the OFDM symbol previous to the one used to transmit the first copy of the P-SCH, whereas the second one is located at the OFDM symbol preceding the one used to transmit the second copy of the P-SCH (see Figure 7). Hence, once detected the S-SCH, the signal is timesynchronized at LTE frame level. Given that only the central 62 subcarriers are employed for synchronization, the receiver can synchronize with no a priori knowledge of the total signal bandwidth.

The P-SCH is constructed from a frequency-domain Zadoff-Chu sequence of length 63 [23]. Among its properties, the ability to detect it even with a frequency offset up to $\pm 7.5 \mathrm{kHz}$ is highlighted. Hence, the time offset can be estimated initially without requiring to perform a frequency synchronization (notice also that both the eNodeB and the GTEC Testbed nodes use GPS-disciplined oscillators; thus, the frequency offset between them is very small). On the other hand, the S-SCH sequence is based on maximum length sequences, known as $M$-sequences [23]. Since the S-SCH is detected after the P-SCH, the P-SCH itself can be used to perform a rough channel response estimation to aid in the detection of the S-SCH [23].
TABLE 3: LTE signal configuration parameters.

\begin{tabular}{lcc}
\hline Parameter & Notation & Value \\
\hline OFDM symbols per frame & $s_{F}$ & 140 \\
Samples per frame & $s_{F}^{S}$ & 153600 \\
Samples per slot & $s_{\text {slot }}$ & 7680 \\
FFT size & $N$ & 1024 points \\
Number of used subcarriers & $N_{u}$ & 600 \\
OFDM symbols per LTE slot & $N_{\text {slot }}$ & 7 \\
Long CP length & $N_{\text {long }}^{\mathrm{CP}}$ & 80 samples \\
Short CP length & $N_{\text {short }}^{\mathrm{CP}}$ & 72 samples \\
Mean CP length & $N^{\mathrm{CP}}$ & $\approx 73.14$ samples \\
\hline
\end{tabular}

Since our environment comprises two different sectors, each of them having a different physical cell ID, we can easily determine both the P-SCH and the S-SCH sequences per sector. Hence, both of them can be found by using a simple correlation, thus acquiring the symbol and LTE frame timing.

3.2. Channel Response Estimation. The wireless channel response between the $\mathrm{eNodeB}$ and the receiver is estimated by taking advantage of the pilot structure defined by LTE. Firstly, an estimate of the discrete-time frequency-domain channel response $\widetilde{H}^{l}[n, \lambda]$ is obtained for each LTE frame $l \geq 0$, where $n=0, \ldots, s_{F}-1$ denotes the OFDM symbol index, with $s_{F}$ being the number of OFDM symbols per LTE frame and $\lambda=-N / 2, \ldots, N / 2-1$ the subcarrier index, with $N$ being the Fast Fourier Transform (FFT) size of the OFDM modulator. We used a Minimum Mean Squared Error (MMSE) channel estimator. Refer to Table 3 for the definition of the constants employed in this section.

Notice that frequency synchronization is skipped since one of the parameters that we are estimating is the Doppler PSD, and this process will be altered due to the corrections introduced by the frequency synchronization algorithms. The estimated channel response in the discrete-time domain for the $l$ th frame, namely, $\widetilde{h}^{l}[n, \kappa]$, is obtained by applying the Inverse Fast Fourier Transform (IFFT) operation to 
the corresponding discrete-time frequency-domain channel response as

$$
\widetilde{h}^{l}[n, \kappa]=\operatorname{IFFT}_{\lambda}\left(\widetilde{H}^{l}[n, \lambda]\right),
$$

where $\operatorname{IFFT}_{\lambda}(\cdot)$ denotes the IFFT operation over $\lambda$ and $\kappa=$ $-N / 2, \ldots, N / 2-1$ is the discretized delay index. Note that the channel seen by the signal comprises subcarriers with indices $\lambda=-N_{u}, \ldots, N_{u}$. However, the MMSE channel estimator provides an estimation of the channel response for the $N$ subcarriers considered (i.e., it also includes the guard subcarriers). This enables us to avoid the spreading effect that would appear if guard subcarriers are set to zero due to the properties of the IFFT.

Since the LTE frames are transmitted in a continuous fashion (i.e., there are no gaps or silent periods between consecutive LTE frames), we can obtain an estimation of the wireless channel response along the train path, namely, $\widehat{h}[n, \kappa]$, by concatenating the estimates of successive LTE frames. However, for doing so, the propagation delay and thus the synchronization points (a synchronization point is defined as the index of the first sample of the acquired signal corresponding to a valid LTE frame) must be taken into account.

The synchronization point for the lth LTE frame (see Section 3.1 for more details about the synchronization procedure), namely, $p^{l}$, can be expressed as a function of the frame index $l$ as

$$
p^{l}=n_{i}+l \cdot s_{F}^{S}+\gamma^{l}
$$

being

$$
\gamma^{l}=\left\lfloor\tau^{l} \cdot f_{s}\right\rfloor+e^{l},
$$

where $n_{i}$ is the index of the initial sample for the first transmitted LTE frame, $s_{F}^{S}$ is the number of samples per LTE frame, $\tau^{l}$ is the propagation delay for the $l$ th frame, which depends on the distance between the receive antenna mounted on the train and the transmit antenna at the eNodeB, $[\cdot\rceil$ denotes the round operation, $f_{s}$ is the sampling frequency, and $e^{l}$ is an integer error term which depends on the actual channel and the noise.

The detected synchronization point, $p$, allows us to compensate for the propagation delay in the estimated channel responses, hence centering the most powerful tap around $\kappa=0$. For the case of two consecutive frames $l$ and $l+1$ being synchronized, if $\Delta \gamma^{l} \triangleq \gamma^{(l+1)}-\gamma^{l} \neq 0$, there will be a discontinuity in the estimated channel responses between the two frames (note that $\Delta \gamma^{l}=p^{(l+1)}-p^{l}-s_{F}^{S}$ ). The continuity can be preserved by accounting for the propagation delay of the received frames. This can be done since the IFFT size in (1) equals the number of subcarriers; thus, the sampling time of the signal and the estimated channel delays match. To preserve the continuity we can follow different strategies.

3.2.1. Synchronizing Only the First Frame. We can take advantage of the OFDM cyclic prefix (CP) and synchronize only the first frame. Then, instead of synchronizing each

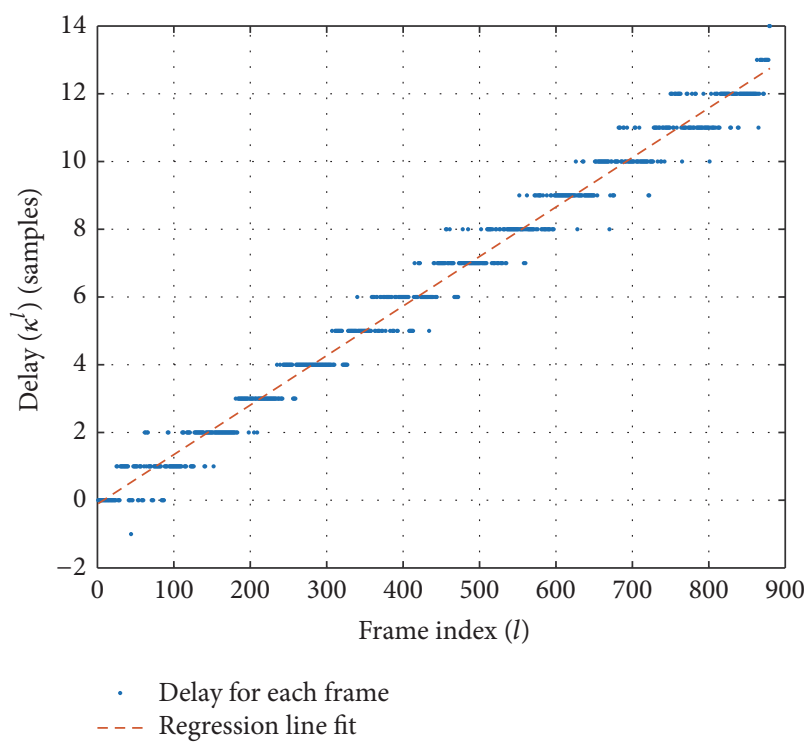

FIGURE 8: Exemplary results of delays $\left(\kappa^{l}\right)$ for a portion of the captured frames.

successive LTE frame (hence obtaining the synchronization points defined in (2)), the synchronization point for the $l$ th frame is obtained as $p_{l}=n_{i}+l \cdot s_{F}^{S}$, without accounting for the propagation delay. This way the overall channel response (i.e., the concatenation of the channel responses for the subframes along the whole path) is defined as

$$
\tilde{h}[n, \kappa]=\widetilde{h}^{l}\left[n-s_{F} \cdot l, \kappa-\kappa^{0}\right], \quad l=\left\lfloor\frac{n}{s_{F}}\right\rfloor,
$$

where $L \cdot\rfloor$ is the floor operator and $\kappa^{0}$ is the delay of the first synchronized frame.

This method is only valid when the propagation delay is relatively small compared to the CP length; otherwise, Intersymbol Interference (ISI) will arise in the signal.

3.2.2. Synchronize Each Frame and Find Relative Delays. When synchronizing all the frames, we obtain the synchronization points defined in (2). The corresponding delay, expressed as the number of samples for the $l$ th frame, namely, $\kappa^{l}$, is

$$
\kappa^{l}=\kappa^{0}+\sum_{m=0}^{l-1} \Delta \gamma^{l}
$$

where $\kappa^{0}$ is the delay (expressed in number of samples) for the first synchronized frame. Therefore, the overall channel response is

$$
\widetilde{h}[n, \kappa]=\widetilde{h}^{l}\left[n-s_{F} \cdot l, \kappa-\kappa^{l}\right], \quad l=\left\lfloor\frac{n}{s_{F}}\right\rfloor .
$$

Figure 8 shows some exemplary results for the obtained delay samples, $\kappa^{l}$, versus the frame number $l$, considering $\kappa^{0}=0$. 
These results correspond to a portion of the captured frames for the case of the train moving away from the eNodeB at a speed of $100 \mathrm{~km} / \mathrm{h}$. We also show the fitting of the data obtained by a robust linear regression [24]. The slope of the curve shows how the propagation delay increases as the frames are received while the train moves away from the eNodeB, thus increasing the distance between transmit and receive antennas.

3.2.3. Hybrid Approach. We can use a hybrid approach from the two above-mentioned methods. Firstly, the acquired signals are grouped in sets of consecutive frames. Secondly, the channel response is estimated for each set as explained in Section 3.2.1. Then, the propagation delays are estimated for each set following a procedure similar to the one in Section 3.2.2.

The initial time (in seconds) corresponding to the estimated channel response $\widetilde{h}[n, \kappa]$ for each value of $n$, namely, $t_{n}$, can be approximated as

$$
t_{n} \simeq n \frac{1}{f_{s}}\left(N+\overline{N^{\mathrm{CP}}}\right)+\widehat{t}_{0}
$$

where $\widehat{t}_{0}$ is the initial time of the estimated channel response for the symbol with $n=0$, and

$$
\overline{N^{\mathrm{CP}}}=\frac{\left(N_{\text {slot }}-1\right) N_{\text {short }}^{\mathrm{CP}}+N_{\text {long }}^{\mathrm{CP}}}{N_{\text {slot }}}
$$

is the mean number of samples of the CP within the LTE frame, where $N_{\text {long }}^{\mathrm{CP}}$ and $N_{\text {short }}^{\mathrm{CP}}$ are the lengths of the long and short LTE CP, and $N_{\text {slot }}$ is the number of OFDM symbols per slot. Notice that the equality in (7) holds if $n \bmod N_{\text {slot }}=0$.

The delay (in seconds) corresponding to the estimated channel response $\widetilde{h}[n, \kappa]$ for each value of $\kappa$, namely $\tau_{\kappa}$, is obtained as

$$
\tau_{\kappa}=\frac{\kappa}{f_{s}}
$$

Note that it is possible to obtain an exact expression for $t_{n}$ as

$$
t_{n}=\frac{1}{f_{s}}\left(\left\lfloor\frac{n}{N_{\text {slot }}}\right\rfloor s_{\text {slot }}+u_{\text {slot }}\left(n \bmod N_{\text {slot }}\right)\right)+\widehat{t}_{0},
$$

where

$$
\begin{aligned}
& u_{\text {slot }}(m) \\
& \quad=\left[N_{\text {long }}^{\mathrm{CP}}+N+\left(N_{\text {short }}^{\mathrm{CP}}+N\right)(m-1)\right]\left(1-\delta_{m, 0}\right)
\end{aligned}
$$

is the index of the starting sample of the $m$ th OFDM symbol within an LTE slot, $s_{\text {slot }}^{S}$ is the number of time-samples per LTE slot, and $\delta_{p, q}$ is the Kronecker delta function defined as

$$
\delta_{i, j}= \begin{cases}1 & \text { if } i=j \\ 0 & \text { if } i \neq j\end{cases}
$$

For many of the estimations performed in the following sections (e.g., see Section 5), it is more convenient to consider a channel with an impulse response starting at delay zero for all $n$, namely, $\widehat{h}[n, \kappa]$. This allows us to consider the same subset of delays (when estimating parameters from the channel (see Section 5), we use a finite number of delays less than $N_{\text {short }}^{\mathrm{CP}}$ ) along the whole path for estimating each desired parameter. Therefore, we estimate the propagation delay for each frame $l$ as the number of samples including a fractional part: namely, $\widehat{\kappa}^{l}=\kappa_{i}^{l}+\kappa_{f}^{l}$, where $\kappa_{i}^{l}$ is the integer part and $\kappa_{f}^{l}$ is the fractional one. Then, we can estimate the channel response for the $l$ th frame correcting the fractional part as

$$
\widehat{h}^{l}[n, \kappa]=\operatorname{IFFT}_{\lambda}^{N}\left(\widetilde{H}^{l}[n, \lambda] \cdot e^{-j 2 \pi(\lambda / N) \kappa_{f}^{l}}\right) .
$$

The integer part is compensated by applying an offset to the delay when obtaining the complete channel response; that is,

$$
\widehat{h}[n, \kappa]=\widehat{h}^{l}\left[n-s_{F} \cdot l, \kappa-\kappa_{i}^{l}\right], \quad l=\left\lfloor\frac{n}{s_{F}}\right\rfloor .
$$

3.3. SNR Estimation. The SNR is estimated considering exclusively the data subcarriers by means of the method described in [1, Section 4.5.2]. Guard subcarriers, Direct Current (DC) subcarrier, and pilot subcarriers are discarded a priori. Hence, the average SNR per data subcarrier is obtained. The SNR estimation is performed by considering the following steps:

(1) Noise samples in the time domain are captured with the transmitter switched off (hence not transmitting any signal).

(2) The captured noise samples in the time domain are then processed as if they were actual data samples: that is, the cyclic prefix is removed, a FFT is performed, and both guard and DC subcarriers are removed.

(3) As a result, $w^{(n, \lambda)}$ noise samples are obtained in the frequency domain, each one corresponding to the $\lambda$ th subcarrier of the $n$th OFDM symbol, where $\lambda \epsilon$ $\mathscr{D}^{(n)}$, with $\mathscr{D}^{(n)}$ being the set of indexes of the data subcarriers for the $n$th OFDM symbol.

(4) All previous steps are repeated when the transmitter is switched on, so $r^{(n, \lambda)}$ data samples (of course affected by noise) are obtained in the frequency domain.

(5) The average SNR for each OFDM symbol is then estimated by averaging out the instantaneous SNR values for the different data subcarriers. Define

$$
\begin{aligned}
& \bar{r}^{(n)}=\frac{1}{\left|\mathscr{D}^{(n)}\right|} \sum_{\lambda \in \mathscr{D}^{(n)}}\left|r^{(n, \lambda)}\right|^{2}, \\
& \bar{w}^{(n)}=\frac{1}{\left|\mathscr{D}^{(n)}\right|} \sum_{\lambda \in \mathscr{D}^{(n)}}\left|w^{(n, \lambda)}\right|^{2},
\end{aligned}
$$


where $\left|\mathscr{D}^{(n)}\right|$ is the cardinality of $\mathscr{D}^{(n)}$, that is, the number of data subcarriers for the $n$th symbol. Then, the SNR for each OFDM symbol is calculated as

$$
\operatorname{SNR}^{(n)}=\frac{\bar{r}^{(n)}-\bar{w}^{(n)}}{\bar{w}^{(n)}} .
$$

\section{Path Loss Estimation}

The path loss is defined as the ratio between the transmitted and the received power and it is expressed in decibels as a function of the distance as [25]

$$
\mathrm{PL}(d)=10 \log _{10} \frac{P_{t}}{P_{r}(d)},
$$

where $\operatorname{PL}(d)$ is the path loss (in $\mathrm{dB}$ ) for a distance $d, P_{t}$ is the transmit power, and $P_{r}(d)$ is the receive power at a distance $d$. In this manuscript, we adopt a simplified logdistance path loss model which allows us to characterize the large-scale path loss as well as the shadow fading variations of the channel response. This model is expressed as in [25]

$$
\begin{aligned}
\widetilde{\mathrm{PL}}(d) & =\overline{\mathrm{PL}}\left(d_{0}\right)+10 \gamma \log _{10}\left(\frac{d}{d_{0}}\right)+X_{\sigma} \\
& =b+10 \gamma \log _{10} d+X_{\sigma},
\end{aligned}
$$

where $\gamma$ is the so-called path loss exponent, $d_{0}$ is a distance relatively close to the transmitter known as "break distance" or "breakpoint," $X_{\sigma}$ is a zero-mean Gaussian random variable with a standard deviation $\sigma$ representing the variations due to the medium and small-scale fading, and $\overline{\mathrm{PL}}\left(d_{0}\right)$ corresponds to the mean path loss at the break distance. The terms depending on $d_{0}$ are reorganized to obtain the last expression in (18).

Using (18) (neglecting $X_{\sigma}$ ), we estimate $b$ and $\gamma$ for each combination of active sector, receive antenna, and measured sector by using least-squares fitting over the energy of the receive signal (note that the low speed measurements, as explained in Section 2.3, are used here). This way we obtain the large-scale fading curves for each scenario. Figures 9 and 10 show the received energy for both sectors when only a single sector was active. Note that only the results for outdoor antenna 1 and indoor antenna 1 are shown in all the plots; the results for the other antennas are similar. For each energy curve, the corresponding fitting is provided. As shown in Figures 9 and 10, for the highest and the lowest considered distance values, the model does not fit well the data due to the high directivity of the transmit antennas. This is because the omnidirectional model assumed for the transmit antennas does not hold in this case. Figures 9 and 10 also show that the antennas serving the north sector cause interference into the south sector and vice versa. This is due to the configuration of the eNodeB antennas.

Given that $X_{\sigma}$ is modeled as a zero-mean Gaussian random variable with standard deviation $\sigma$, we subtract the obtained fittings from the received energies to estimate such a standard deviation $\sigma$. Figures 11 and 12 show the estimated cumulative probabilities of $X_{\sigma}$ when only a single sector

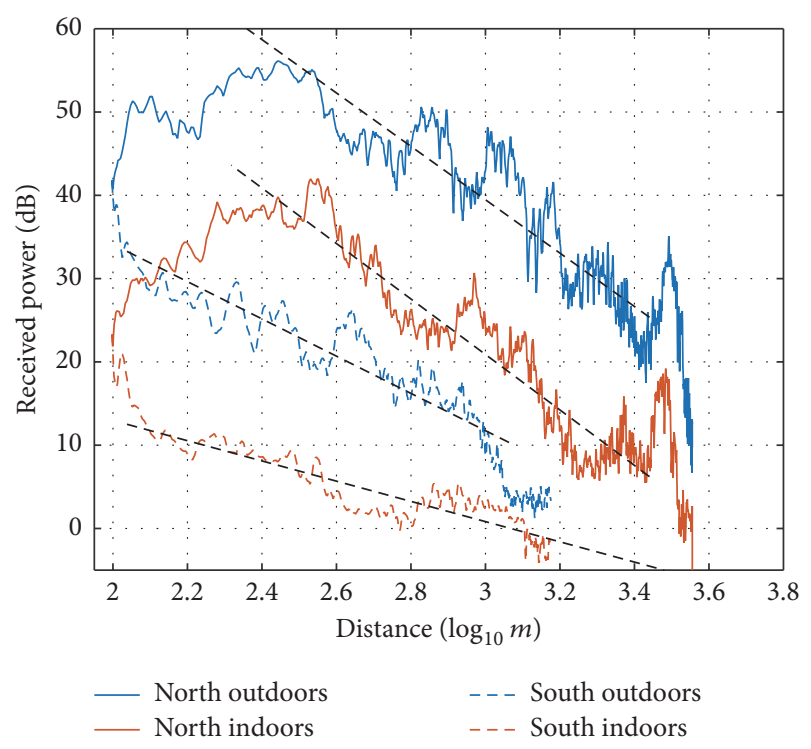

FIGURE 9: Received energy and least-squares fitting along both north and south sectors for the receive outdoor antenna 1 and the receive indoor antenna 1 . Only the antennas serving the north sector were transmitting.

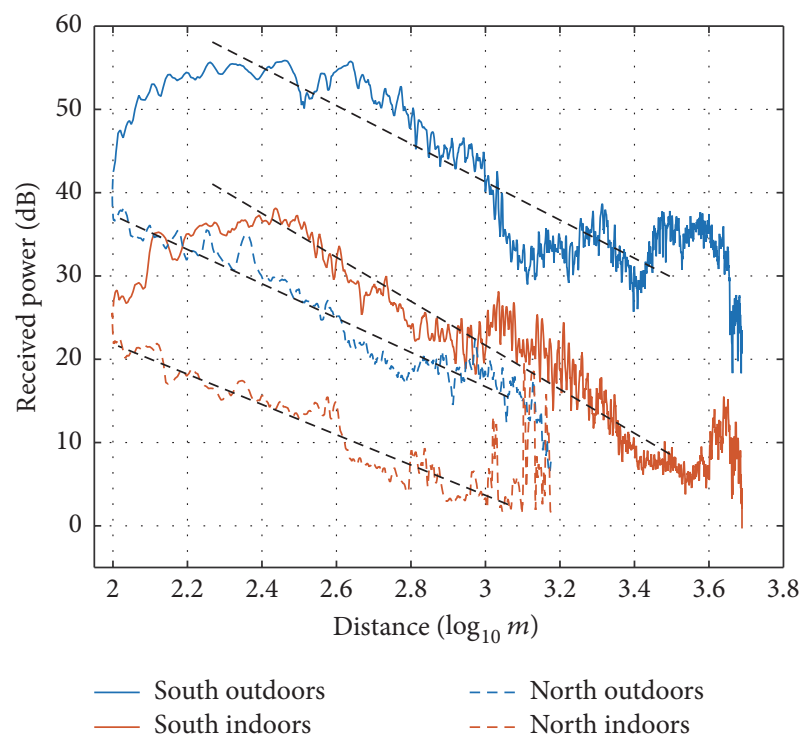

FIGURE 10: Received energy and least-squares fitting along both north and south sectors for the receive outdoor antenna 1 and the receive indoor antenna 1 . Only the antennas serving the south sector were transmitting.

(north or south) is active. We also plot the theoretical cumulative probabilities of zero-mean Gaussian distributions with the standard deviation $\sigma$ estimated for each case. We can see that the fitting between the estimated and theoretical curves is very good. Finally, Table 4 shows the corresponding path loss parameters $(\gamma, b$, and $\sigma)$ estimated from the measurement data.

As seen in Table 4, the path loss exponent is very similar for all cases (both sectors as well as indoor and 

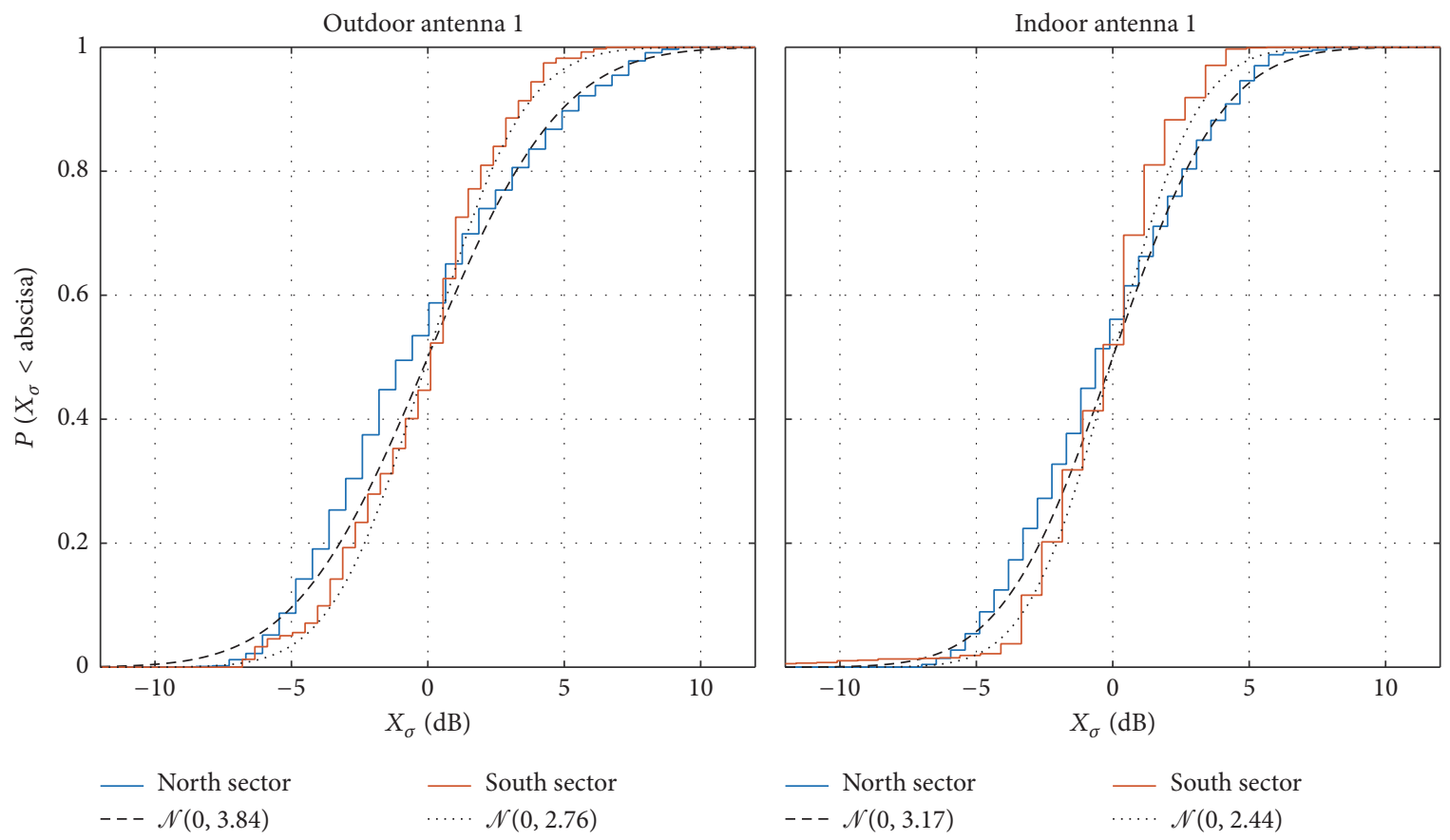

FIGURE 11: Cumulative probabilities for receive outdoor antenna 1 and indoor antenna 1 along both north and south sectors. Only the antennas serving the north sector were transmitting.

TABLE 4: Path loss parameters $(\gamma, b$, and $\sigma)$ estimated for both north $(\mathrm{N})$ and south $(\mathrm{S})$ sectors and for each of the four receive antennas: two of them are placed inside the train car ("indoor ant. 1" and "indoor ant. 2") and the other two are placed outdoors, on the train roof ("outdoor ant. 1" and "outdoor ant. 2"). The results are obtained considering a single active sector (north or south), that is, the sector whose antennas are transmitting. The column TX specifies the active sector, whereas the column RX denotes the sector along which the measurements are performed.

\begin{tabular}{ccccccccccccccc}
\hline \multirow{2}{*}{ TX } & RX & \multicolumn{3}{c}{ Outdoor ant. 1 } & \multicolumn{3}{c}{ Outdoor ant. 2 } & \multicolumn{3}{c}{ Indoor ant. 1 } & \multicolumn{3}{c}{ Indoor ant. 2 } \\
& & $\gamma$ & $b$ & $\sigma$ & $\gamma$ & $b$ & $\sigma$ & $\gamma$ & $b$ & $\sigma$ & $\gamma$ & $b$ \\
\hline \multirow{2}{*}{$\mathrm{N}$} & $\mathrm{N}$ & 3.20 & 135.61 & 3.84 & 2.53 & 113.34 & 4.08 & 3.33 & 120.88 & 3.18 & 3.17 & 115.93 & 2.85 \\
& $\mathrm{~S}$ & 2.23 & 78.63 & 2.76 & 1.80 & 64.94 & 1.83 & 1.20 & 36.94 & 4.38 & 0.74 & 26.75 & 1.06 \\
$\mathrm{~S}$ & $\mathrm{~N}$ & 2.06 & 78.44 & 1.91 & 2.23 & 78.70 & 1.78 & 1.82 & 58.16 & 2.09 & 1.89 & 60.96 & 1.56 \\
& $\mathrm{~S}$ & 2.29 & 110.00 & 3.74 & 2.32 & 103.36 & 1.68 & 2.64 & 100.89 & 2.70 & 2.58 & 98.91 & 2.39 \\
\hline
\end{tabular}

outdoor receive antennas). However, a large penetration loss is observed for the indoor receiver. This justifies the utilization of a relay scheme for provisioning services to the users in a practical deployment. Hence, for the rest of the analysis, we consider exclusively the external link (eNodeB to outdoor antennas).

\section{Estimation of Channel Condensed Parameters}

The so-called condensed parameters allow us to characterize other effects of the channel different than the path loss. In this section, we consider condensed parameters like $K$-Factor, PDP, delay spread, and Doppler PSD.

5.1. K-Factor. The evaluated scenario can be classified as rural area and exhibits a strong Line-of-Sight (LoS) component during most of the train trajectory. Thus, one of the channel paths presents a predominant power with respect to the others. Due to this, the fluctuations of the path gain follow a Rice distribution. The Rice distribution is parametrized by the power ratio of the fixed and fluctuating components of the path loss, namely, the $K$-Factor.

The instantaneous values of the $K$-Factor are calculated according to the method proposed by Greenstein et al. in [26]. More specifically, [26, Eq. (9)] was evaluated for the experimental data, taking into account the tap corresponding to the LoS component.

Figures 13 and 14 show the instantaneous values of the $K$ Factor expressed in decibels versus the KP for the train speeds of $100 \mathrm{~km} / \mathrm{h}$ and $200 \mathrm{~km} / \mathrm{h}$, respectively. Smoothed values of the $K$-Factor by using a sliding window are also plotted in such figures. The obtained results show that the $K$-Factor is noticeably higher than zero, which is coherent with the hypothesis of the existence of a strong LoS component. Only at certain positions along the train trajectory the $K$-Factor exhibits deep local minimum values (e.g., at KPs $96.6 \mathrm{~km}$ and $98.4 \mathrm{~km}$ ), which are caused by reflecting obstacles along the 

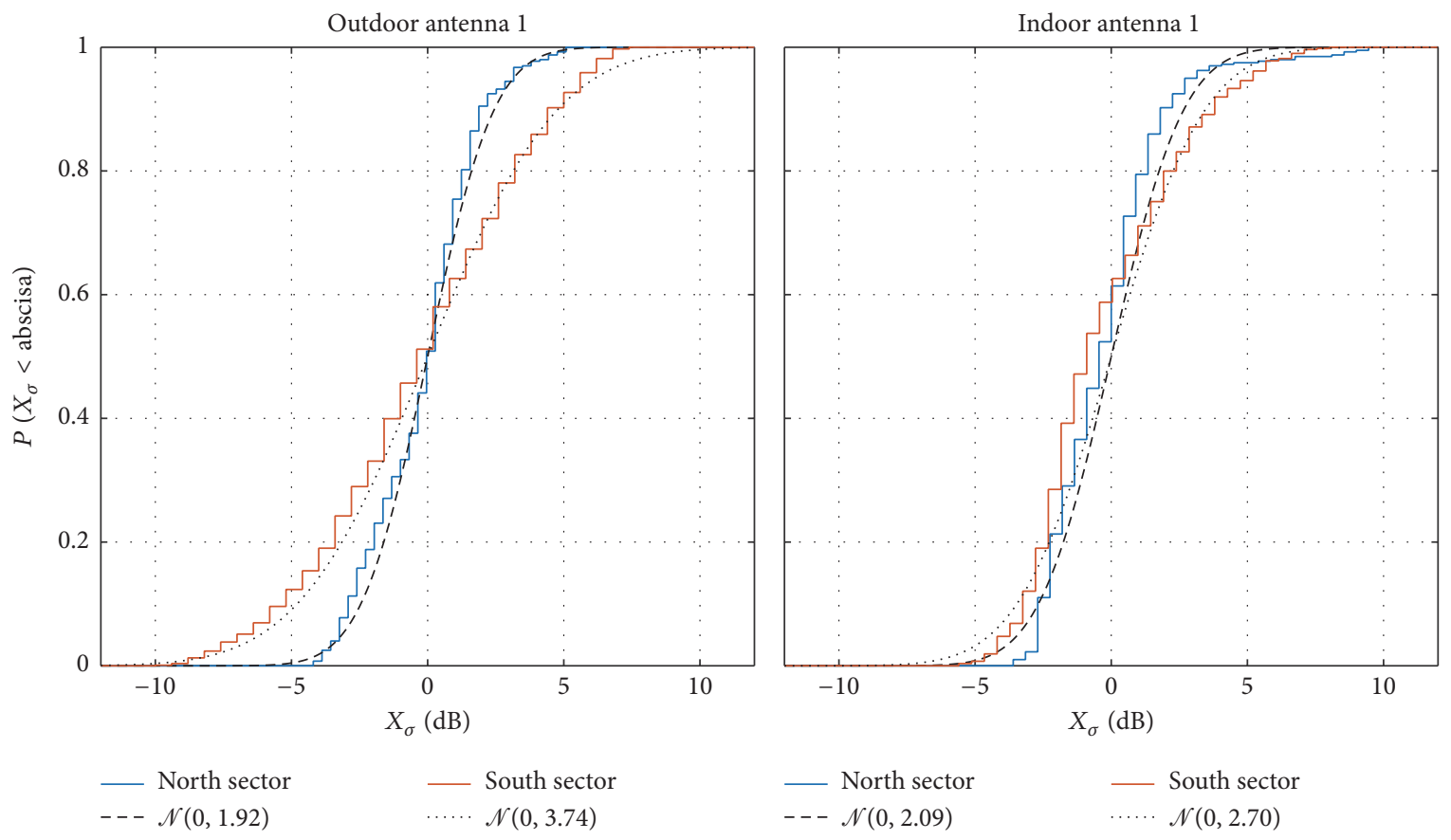

FIGURE 12: Cumulative probabilities for receive outdoor antenna 1 and indoor antenna 1 along both north and south sectors. Only the antennas serving the south sector were transmitting.

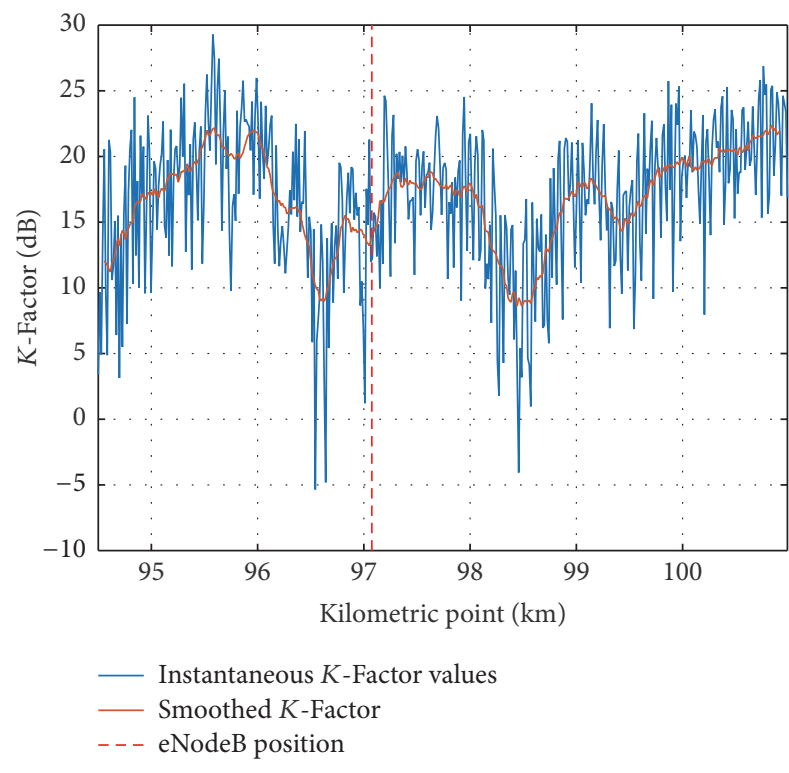

FIGURE 13: Estimated $K$-Factor for outdoor antenna 1 for the train moving at $100 \mathrm{~km} / \mathrm{h}$ from the north sector to the south one. Both sectors are active. The results of averaging out the instantaneous values by means of a sliding window are also shown.

train path (see Section 6 for more details). It can be also seen that the trend of the $K$-Factor does not change significantly with the train speed.

5.2. Power Delay Profile. A parameter typically used to analyze how much energy arrives at the receiver with a certain delay is the PDP. The PDP around the $n$th OFDM symbol can

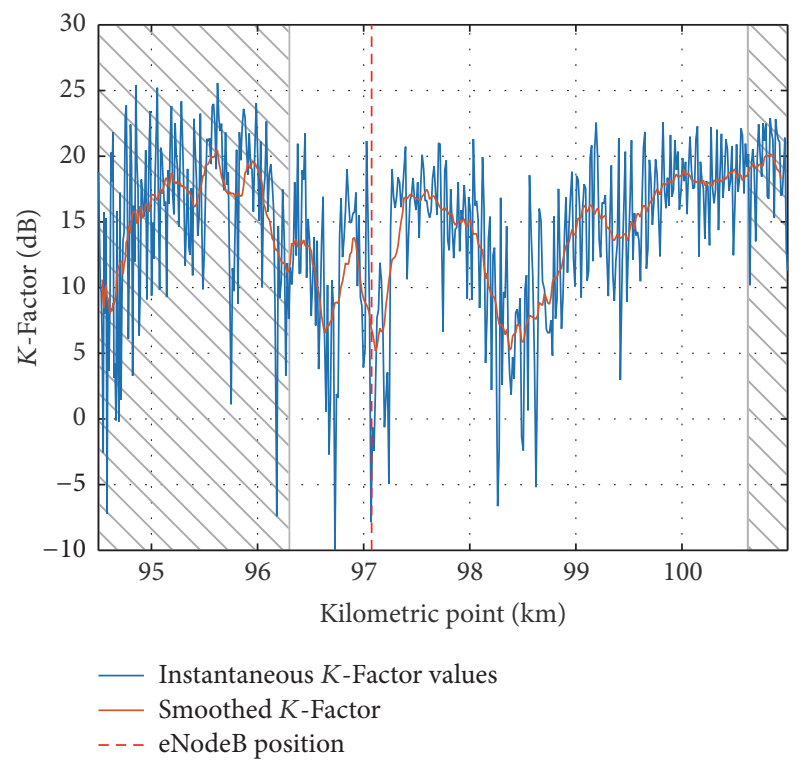

FIGURE 14: Estimated $K$-Factor for outdoor antenna 1 for the train moving at $200 \mathrm{~km} / \mathrm{h}$ from the north sector to the south one. Both sectors are active. The overimposed pattern of gray diagonal lines denotes the areas where the train speed was not constant. The results of averaging out the instantaneous values by means of a sliding window are also shown.

be obtained from a complex-valued channel time response estimate as in [27]

$$
P[n, \kappa]=\frac{1}{2 \alpha+1} \sum_{m=n-\alpha}^{n+\alpha}|\widetilde{h}[m, \kappa]|^{2},
$$




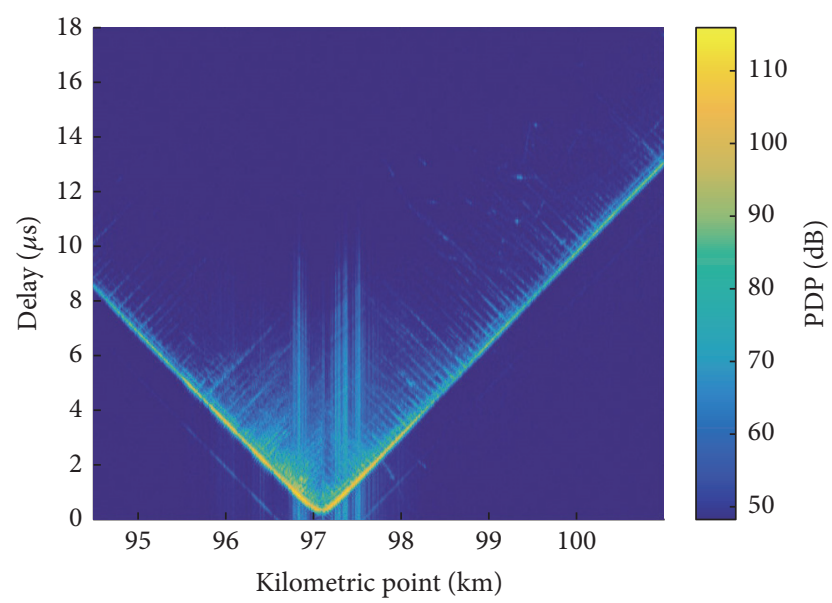

FIGURE 15: Estimated PDP for outdoor antenna 1 for the train moving at $100 \mathrm{~km} / \mathrm{h}$ from the north sector to the south one. Both sectors are active.

where $\alpha$ adjusts the length of the considered segment of the time response (the considered segment includes the time responses for the OFDM symbols whose indexes are $n-$ $\alpha, \ldots, n+\alpha)$.

Figures 15 and 16 show the estimated PDP for the train moving at $100 \mathrm{~km} / \mathrm{h}$ and $200 \mathrm{~km} / \mathrm{h}$, respectively, with both sectors being active. The presented results are obtained by evaluating (19) for equally spaced OFDM symbols. Note that the shape of the PDP curves accounts for the propagation delay, where the values with the lowest delay are the ones corresponding to the KPs closer to the transmit antenna. Note also that the magnitude of the PDP decreases with the distance to the eNodeB antennas.

By comparing Figures 15 and 16, we can conclude that the PDP is independent from the train speed. It is important to note that the longer PDP responses around the eNodeB are caused by the energy spreading effect of the IFFT. This effect is not so noticeable for other KPs since their power is much lower.

Results corresponding to the train moving at $100 \mathrm{~km} / \mathrm{h}$ are shown in Figure 17 when the propagation delay is subtracted; that is, we evaluate (19) by using $\widehat{h}[n, \kappa]$ instead of $\widetilde{h}[n, \kappa]$ for equally spaced OFDM symbols. Furthermore, the values obtained per frame were normalized; hence, the magnitude obtained does not depend on the distance to the transmit antenna. This way we can perform a fair comparison between the PDP results for different KPs, not taking into account the energy spreading effect of the IFFT. It can be seen that PDP responses are noticeable longer for some areas (e.g., at KPs $96.6 \mathrm{~km}$ and $98.4 \mathrm{~km}$ ), which is caused by reflecting obstacles along the train path (see Section 6 for more details).

It has been shown that under some specific circumstances the error probability due to delay dispersion is proportional to the normalized second-order central moment of the PDP, namely, the RMS delay spread [27]. RMS delay spread can be calculated as

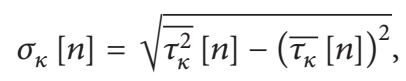

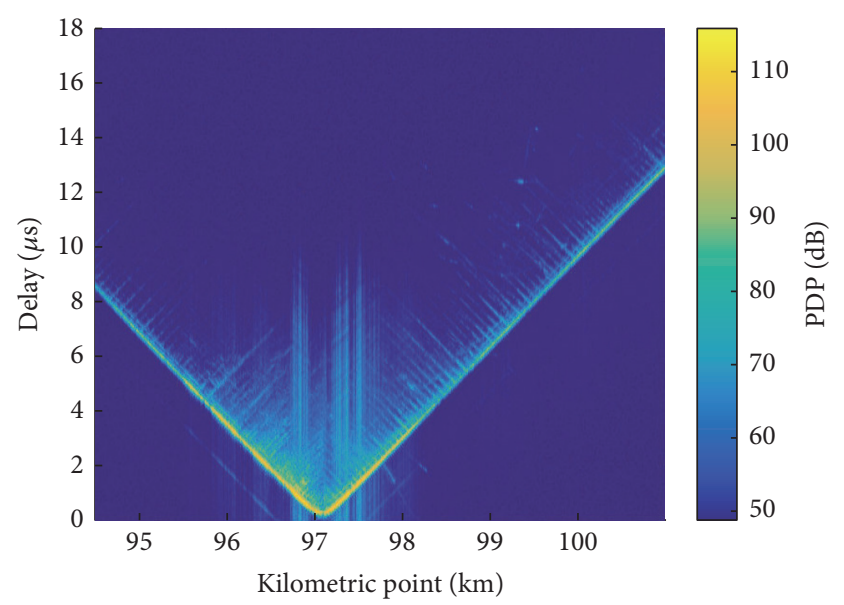

FIGURE 16: Estimated PDP for outdoor antenna 1 for the train moving at $200 \mathrm{~km} / \mathrm{h}$ from the north sector to the south one. Both sectors are active.

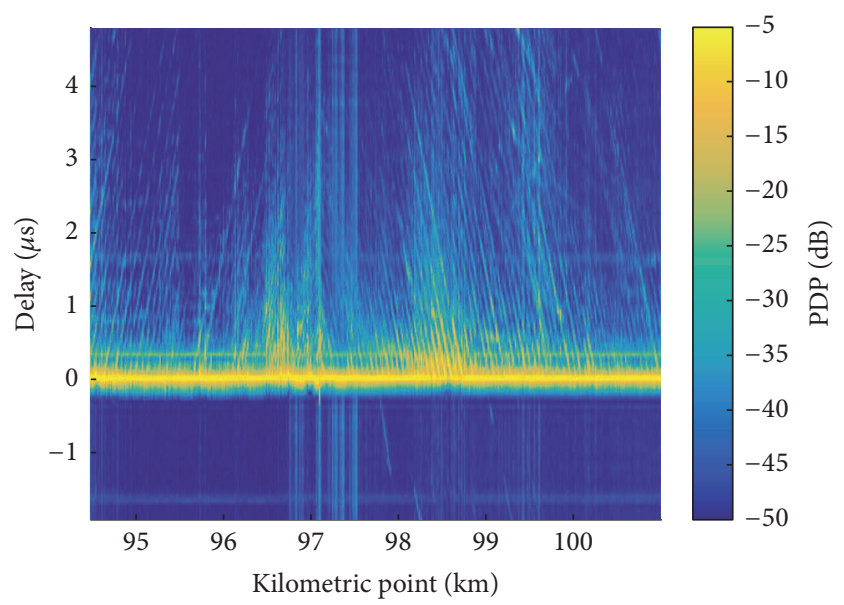

FIGURE 17: Normalized PDP for outdoor antenna 1 for the train moving at $100 \mathrm{~km} / \mathrm{h}$ from the north sector to the south one, not taking into account the propagation delay. Both sectors are active.

where the second moment of $\tau_{\kappa}$ is given by

$$
\overline{\tau_{\kappa}^{2}}[n]=\frac{\sum_{\kappa} P[n, \kappa] \tau_{\kappa}^{2}}{\sum_{\kappa} P[n, \kappa]}
$$

and the first one is obtained as

$$
\overline{\tau_{\kappa}}[n]=\frac{\sum_{\kappa} P[n, \kappa] \tau_{\kappa}}{\sum_{\kappa} P[n, \kappa]} .
$$

Figures 18 and 19 show the RMS delay spread for equally spaced OFDM symbols for the train moving at $100 \mathrm{~km} / \mathrm{h}$ and $200 \mathrm{~km} / \mathrm{h}$, respectively. The plots also include the results of smoothing out the instantaneous RMS delay spread values by means of a sliding window, as well as the mean delay spread for the whole path followed by the train. In Figures 18 and 19, we can see that the delay spread results are coherent regardless of the considered speed. Results shown in Figures 18 and 19 are congruent with those in Figure 17, showing a larger delay spread for the areas with more reflections. 


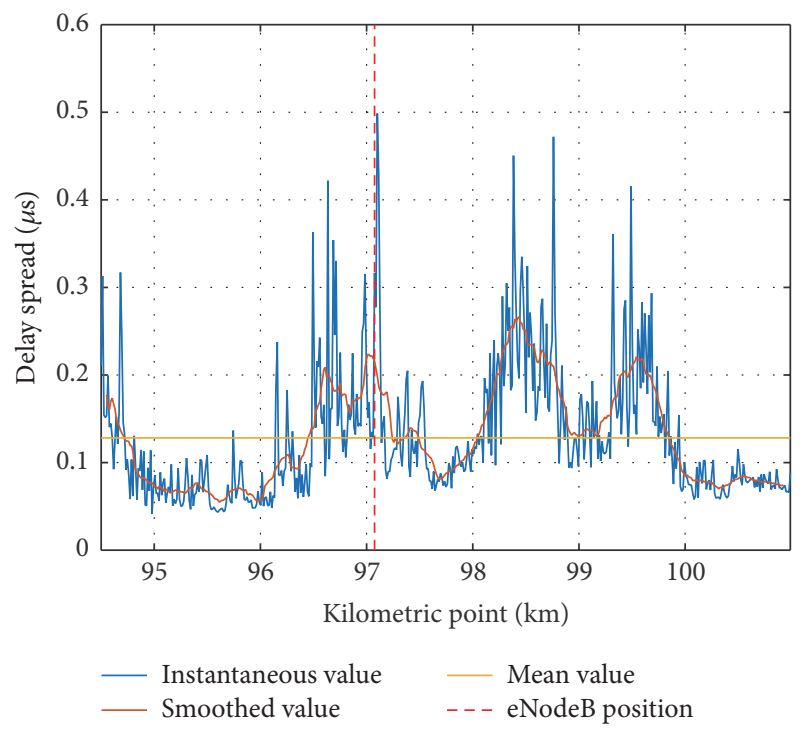

FIGURE 18: Estimated RMS delay spread for outdoor antenna 1 for the train moving at $100 \mathrm{~km} / \mathrm{h}$ from the north sector to the south one. Both sectors are active. A curve corresponding to smoothed instantaneous values by means of a sliding window are also shown, as well as the mean delay spread for the whole path.

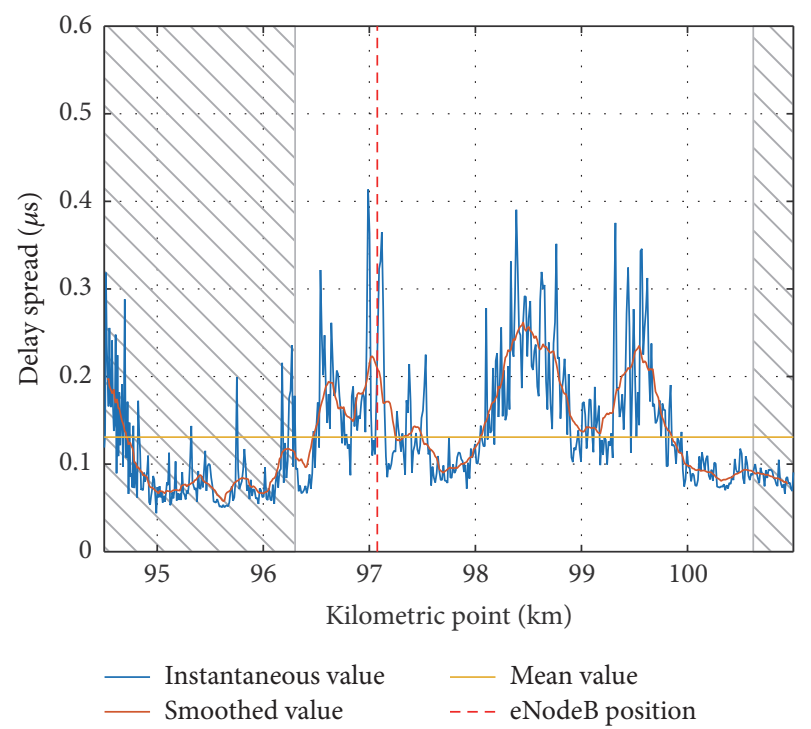

FIGURE 19: Estimated RMS delay spread for the train moving at $200 \mathrm{~km} / \mathrm{h}$ from the north sector to the south one. Both sectors are active. The overimposed pattern of gray diagonal lines denotes the areas where the train speed was not constant. Smoothed instantaneous values by means of a sliding window are also shown, as well as the mean delay spread for the whole path.

5.3. Doppler Power Spectral Density. The Doppler Power Spectral Density is related to the Angle of Arrivals (AoAs) of the multipath components and the direction of movement of the receiver. Doppler PSD around the $n$th OFDM symbol for the delay index $\kappa$, namely, $S[n, \kappa, \theta]$, can be obtained as the FFT of the autocorrelation of a certain segment of the time response of the channel centered at time index $n$. Considering

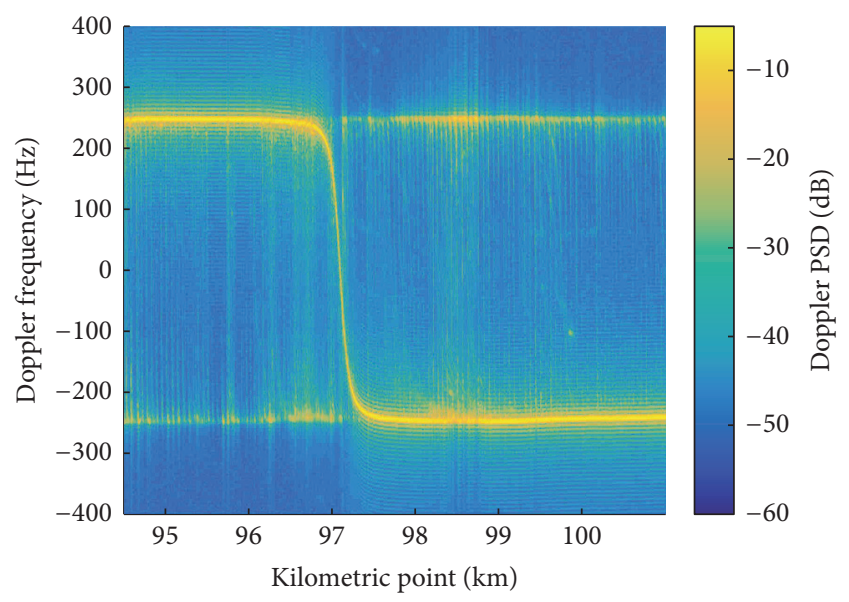

FIgURE 20: Doppler Spectrum for outdoor antenna 1 for the train moving at $100 \mathrm{~km} / \mathrm{h}$ from the north sector to the south one, with both sectors being active.

the estimated channel response $\widehat{h}[n, \kappa]$, the autocorrelation values are obtained as

$$
R_{\widehat{h}}[n, \kappa, \lambda]= \begin{cases}\sum_{m=n}^{n+2 \alpha-\lambda} \widehat{h}[m+\lambda, \kappa] \widehat{h}^{*}[m, \kappa], & \lambda \geq 0 \\ \sum_{m=n}^{n+2 \alpha+\lambda} \widehat{h}[m, \kappa] \widehat{h}^{*}[m+\lambda, \kappa], & \lambda<0,\end{cases}
$$

where $\lambda=-2 \alpha, \ldots, 2 \alpha$ is the lag of the autocorrelation and $\alpha$ adjusts the length of the considered segment of the channel time response. Then, the Doppler scattering function [27] can be written as

$$
S[n, \kappa, \theta]=\operatorname{FFT}_{\lambda}\left\{R_{\widehat{h}}[n, \kappa, \lambda]\right\},
$$

where $\mathrm{FFT}_{\lambda}\{\cdot\}$ denotes the FFT of the argument with respect to the autocorrelation lag $\lambda$ and $\theta$ is the discretized Doppler frequency index, being the corresponding Doppler frequency

$$
v_{\theta}=\theta \frac{f_{s}}{N+\overline{N^{\mathrm{CP}}}} \frac{N_{\text {corr }}}{N_{D}}
$$

where $N_{\text {corr }}=4 \alpha+1$ is the number of samples of the autocorrelation, $N_{D}$ is the size of the FFT, and $\theta=$ $-N_{D} / 2, \ldots, N_{D} / 2-1$. Note that $\left(N+\overline{N^{\mathrm{CP}}}\right) / f_{s}$ is the mean duration of an OFDM symbol. The Doppler PSD $\bar{S}$ is obtained after averaging out the Doppler scattering function $S$ over $\kappa$ values as

$$
\bar{S}[n, \theta]=\frac{1}{|\mathscr{K}|} \sum_{\kappa \in \mathscr{K}} S[n, \kappa, \theta],
$$

where $\mathscr{K}$ denotes the set of considered values for $\kappa$.

Figures 20 and 21 show the normalized Doppler PSD for equally spaced OFDM symbols when the train moves at $100 \mathrm{~km} / \mathrm{h}$ and $200 \mathrm{~km} / \mathrm{h}$, respectively, obtained as indicated in (26). As expected, the module of the maximum Doppler value is related to the train speed as

$$
\left|f_{\max }\right| \approx \frac{|v| f_{c}}{c_{0}}
$$




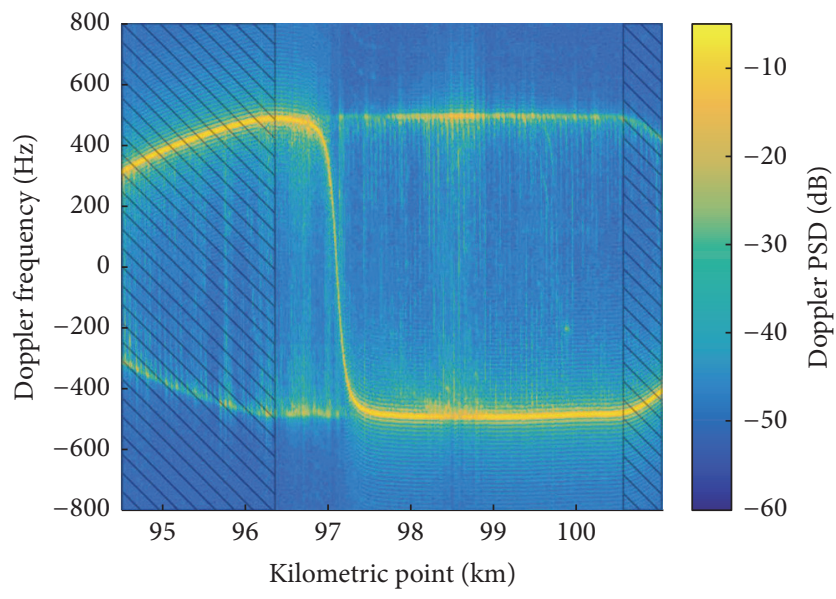

FIGURE 21: Doppler Spectrum for outdoor antenna 1 for the train moving at $200 \mathrm{~km} / \mathrm{h}$ from the north sector to the south one, with both sectors being active. The overimposed pattern of gray diagonal lines denotes the areas where the train speed was not constant.

where $f_{\max }$ denotes the maximum Doppler frequency, $v$ the velocity of the train, and $f_{c}=2.6 \mathrm{GHz}$ the carrier frequency. This brings us to maximum Doppler PSD values of approximately $240.7 \mathrm{~Hz}$ and $480.5 \mathrm{~Hz}$ for train speeds of $100 \mathrm{~km} / \mathrm{h}$ and $200 \mathrm{~km} / \mathrm{h}$, respectively. The sign of the instantaneous Doppler PSD values depends on the direction of the velocity vector of the train with respect to the transmitter. This causes the effect shown in Figures 20 and 21, where the Doppler PSD values concentrate around $\pm\left|f_{\max }\right|$ and change abruptly when the train passes by in front of the eNodeB antennas.

When the train is in the north sector (i.e., it approaches the eNodeB), the Doppler PSD is maximum in $+\left|f_{\max }\right|$. Conversely, when the train is in the south sector (i.e., moving away from eNodeB), the Doppler PSD reaches its maximum at $-\left|f_{\max }\right|$. This is coherent with the measurement environment, which features a strong LoS component. However, significant reflections in $-\left|f_{\max }\right|$ for the north sector (conversely, values in $+\left|f_{\max }\right|$ for the south sector) can be seen, which are caused by obstacles along the train path (see Section 6 for more details).

\section{Particularities of the Measured Train Scenario}

In the previous section, we showed different condensed parameters along the whole train trajectory. However, since the total distance shown is relatively large, small details cannot be appreciated. In this section, we show and discuss the PDP and the Doppler PSD for three specific segments of the train trajectory, each of them being $250 \mathrm{~m}$ long, which are as follows:

(i) Segment (a): KPs between 98.375 and 98.625. There are strong reflections in this area, as shown by the Doppler results in Figures 20 and 21. Also the $K$ Factor achieves the lowest values and the delay spread the highest ones in this area (see Figures 13 and 14 for the $K$-Factor and Figures 18 and 19 for the delay spread). This supports the hypothesis of a weaker LoS condition.

(ii) Segment (b): KPs between 98.875 and 99.125. In this area, the $K$-Factor and the delay spread take values close to the mean ones shown in Figures 13 and 14 and in Figures 18 and 19, respectively.

(iii) Segment (c): KPs between 100.25 and 100.5. In this area, the $K$-Factor achieves the highest values and the delay spread the lowest ones (see Figures 13 and 14 for the $K$-Factor and Figures 18 and 19 for the delay spread).

Figure 22 shows the Doppler Spectrum for the three aforementioned segments when the train moves at $100 \mathrm{~km} / \mathrm{h}$. For all the segments shown, the train moves away from the eNodeB within the south sector; therefore, the LoS Doppler component is located approximately at $-\left|f_{\max }\right|=-240.7 \mathrm{~Hz}$ (see Section 5.3). Furthermore, a noticeable reflection component can be seen in the opposite frequency, $+\left|f_{\max }\right|=$ 240.7 Hz. In addition, abrupt transitions in the Doppler from $-\left|f_{\max }\right|$ to $+\left|f_{\max }\right|$ occur periodically. These transitions in the Doppler are similar to the ones occurring when the train passes by in front of the eNodeB antennas (see Figures 20 and 21). In fact, these abrupt changes in the Doppler are due to the nearby objects reflecting the signal. More specifically, the masts supporting the overhead line (see Figure 26(b)) are the origin of the stronger reflections. It can be seen that the distance between consecutive masts (see Figure 26(a)) is coherent with the separation between consecutive Doppler transitions shown in Figure 22. Finally, it is worth noting that the magnitude of the reflected components with respect to the LoS component depends on the position of the train along the path. This is related both to the distance and the angle with respect to the transmit antenna.

Figure 23 shows the normalized PDP for the three segments, verifying the existence of reflection components coherent with those shown in Figure 22. The PDP figures clearly show that the reflected component associated with each mast decreases its delay with respect to the LoS component (and increases its power) when the train moves towards to the mast. The magnitude of the reflected components with respect to the LoS one is coherent with the relationship between the reflected component and the LoS one shown in Figure 22. For example, the largest reflections in the PDP are obtained for the segment (a). Coherently, the magnitude of the reflections with respect to the LoS component in Figure 22 is also maximum for the segment (a).

It is important to notice that the described effects are independent of the train speed. Figure 25 shows the normalized Doppler PSD and the normalized PDP for the segment (a) when the train moves at $200 \mathrm{~km} / \mathrm{h}$.

Figure 24 shows the estimated Doppler PSD for the three considered segments. Note that the regular oscillations in these results are a manifestation of the Gibbs phenomenon, after evaluating (24), due to the use of time-limited signals to calculate (23). In agreement with Figure 22, the maximum of the Doppler PSD is located in $-\left|f_{\max }\right|$ (due to the contribution of the main path). For the segments with stronger reflected components, a relevant peak is also appreciated in 


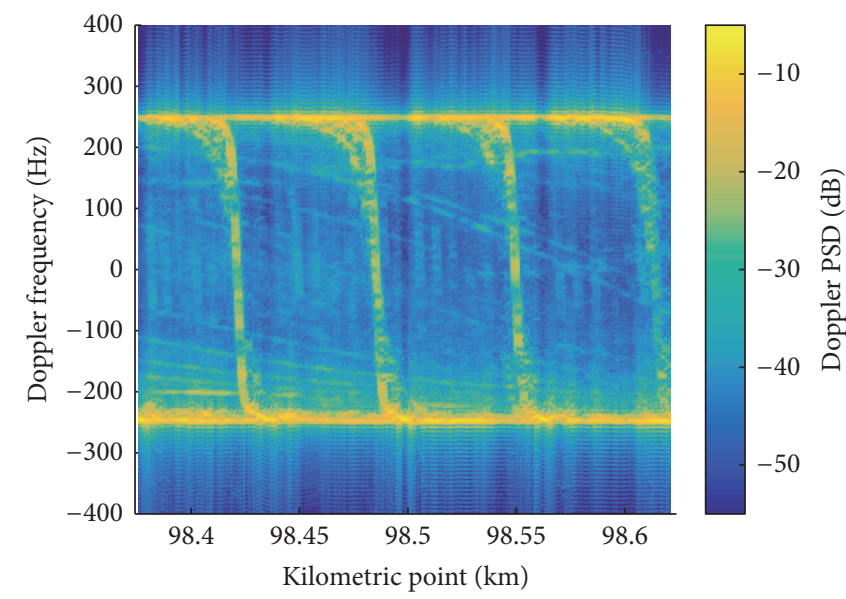

(a) Doppler Spectrum for the train moving at $100 \mathrm{~km} / \mathrm{h}$ along the segment (a)

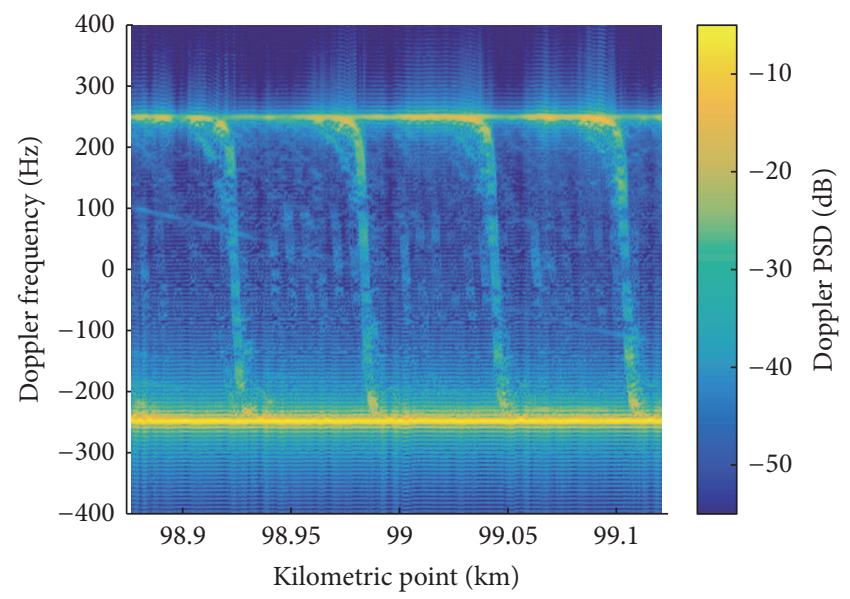

(b) Doppler Spectrum for the train moving at $100 \mathrm{~km} / \mathrm{h}$ along the segment (b)

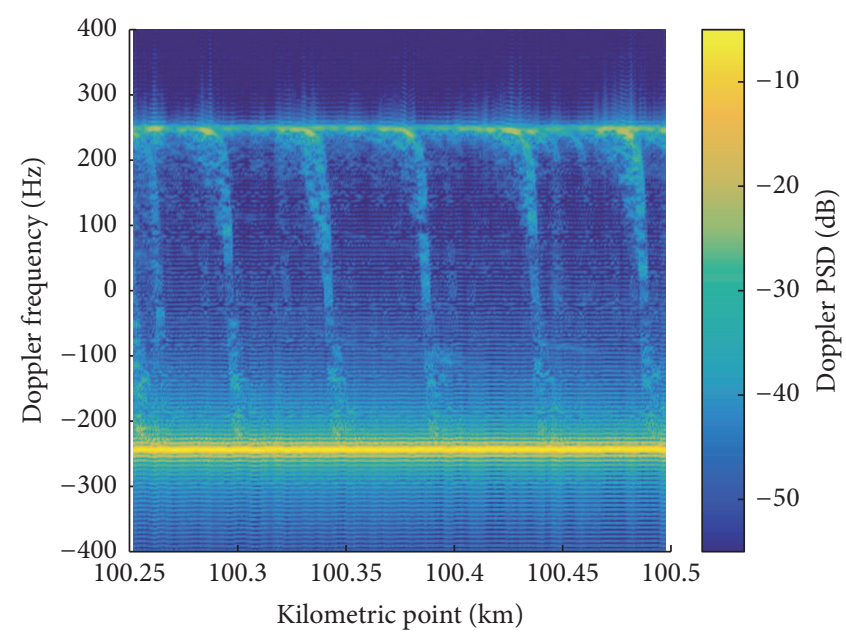

(c) Doppler Spectrum for the train moving at $100 \mathrm{~km} / \mathrm{h}$ along the segment

(c)

FIGURE 22: Doppler Spectrum for outdoor antenna 1 for the train moving at $100 \mathrm{~km} / \mathrm{h}$ along the segments (a), (b), and (c).

$+\left|f_{\max }\right|$. The magnitude of the peak with respect to the main component is reduced for the cases with lower reflections. For other frequencies, the Doppler values are distributed approximately according to a "U" shape. This suggests that, although there is a strong LoS in the railway environment, reflections caused by railway infrastructure elements may lead to a Doppler PSD with noticeable components in other frequencies, similar to the Jakes spectrum [28]. It is worth noting that the condensed parameters are affected by the reflections, as they noticeably change their respective trends in the areas where the reflections are stronger (e.g., segment (a)). Due to this, we can expect severe effective link SNR degradation for such areas.

Note that the obtained results match those shown in [15], obtained for a similar test environment. Finally, Figure 25(c) shows the estimated Doppler PSD for the segment (a) when the train moves at $200 \mathrm{~km} / \mathrm{h}$. The obtained results are coherent with those corresponding to a moving speed of $100 \mathrm{~km} / \mathrm{h}$.

\section{Conclusions}

In this paper, we provided a complete and detailed characterization of the downlink (Long Term Evolution (LTE) Frequency-Division Duplex (FDD) for the $10 \mathrm{MHz}$ profile) between a commercial LTE Evolved NodeB (eNodeB) and a train moving at high velocities along a track in commercial operation in a rural area in Spain. Instead of using an available eNodeB from a general-purpose mobile network, we installed an eNodeB for our measurements at the same location where a GSM for Railways (GSM-R) base station is deployed, thus being able to control all the parameters of the eNodeB. The eNodeB served two sectors using two antennas per sector from different antenna panels installed in the same tower as the GSM-R antennas. We also considered two links: the one between the eNodeB and the antennas placed outdoors on the train roof and the direct link between the eNodeB and a receiver inside the train. With respect to our hardware, the GTEC Testbed nodes were employed as receivers to acquire 


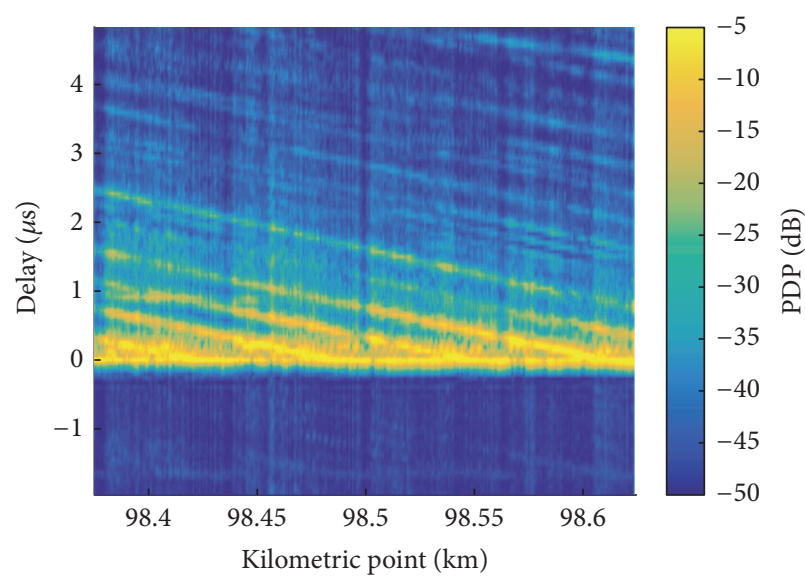

(a) Normalized PDP for the train moving at $100 \mathrm{~km} / \mathrm{h}$ along segment (a)

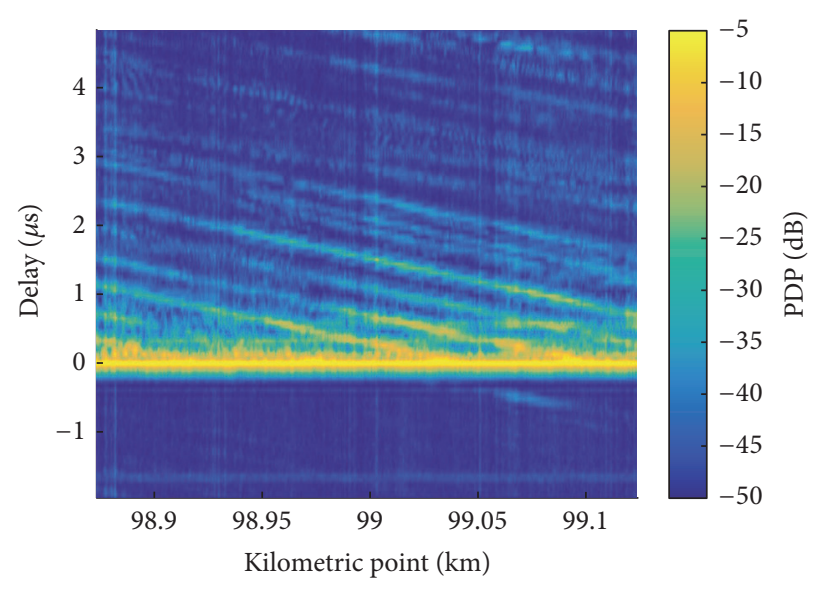

(b) Normalized PDP for the train moving at $100 \mathrm{~km} / \mathrm{h}$ along segment (b)

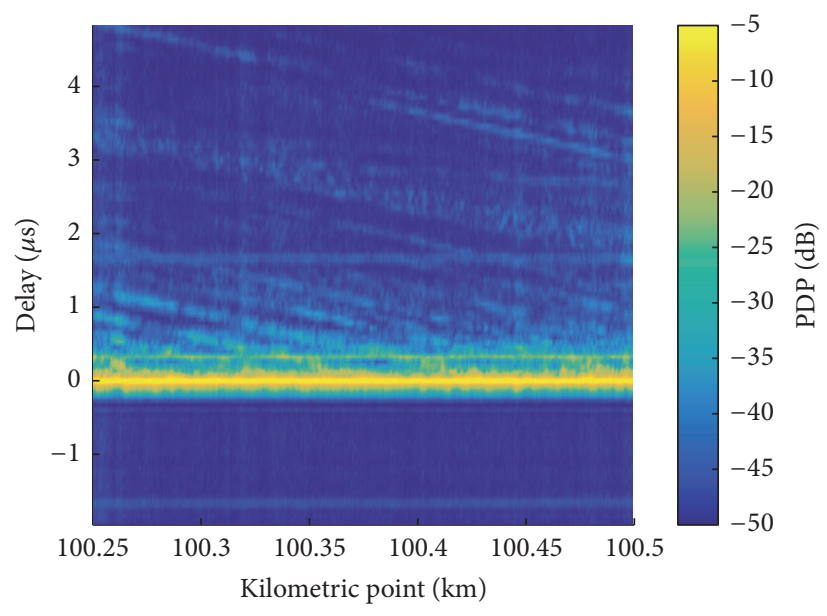

(c) Normalized PDP for the train moving at $100 \mathrm{~km} / \mathrm{h}$ along segment (c)

FIGURE 23: Normalized PDP for outdoor antenna 1 for the train moving at $100 \mathrm{~km} / \mathrm{h}$ along segments (a), (b), and (c). The propagation delay is not taken into account.

the transmit data and were equipped with GPS modules with a twofold purpose: georeferencing the acquired data and minimizing the frequency offset between the eNodeB and the receivers. Regarding the measurement procedure, different train speeds were considered, also with one or both sectors active. A high repeatability level was observed for different train passes with identical configuration parameters, thus enabling us to compare the results at different speeds consistently. Furthermore, high-resolution results were obtained from our measurements, since we continuously captured the signal at the receiver during the whole measurement campaign.

The aforementioned downlink characterization consisted in assessing the path loss, the Signal to Noise Ratio (SNR), the $K$-Factor, the Power Delay Profile (PDP), the delay spread, and the Doppler Power Spectral Density (PSD) for different train speeds. Furthermore, the estimation procedure for each aforementioned parameter was fully described mathematically, highlighting the drawbacks and pitfalls to be considered for the different analysis methods. In particular, the path loss was estimated from the received energy. Assuming a simplified log-distance model, we estimated the path loss exponent and variance of the medium- and small-scale fading. Although the path loss exponent was very similar in all cases (both sectors as well as indoor and outdoor receive antennas), a large penetration loss was observed for the indoor receiver. This would justify the utilization of a relay scheme for provisioning services to the users in a practical deployment. Therefore, we limited the rest of the analysis to the external link (eNodeB to outdoor antennas).

The results corresponding to the $K$-Factor show that a dominant Line-of-Sight (LoS) component was present along the track and that its trend does not depend on the train speed. The same conclusions are obtained from the PDP results, which are also congruent with those obtained for the Root Mean Square (RMS) delay spread. With respect to the Doppler PSD, the largest magnitudes correspond approximately to the frequencies $\pm 240.7 \mathrm{~Hz}$ and $\pm 480.5 \mathrm{~Hz}$ for train speeds of 100 and $200 \mathrm{~km} / \mathrm{h}$, respectively, which is consistent with the presence of a strong LoS component. The sign of the Doppler PSD changes abruptly when the train passes by in front of the transmit antennas. 


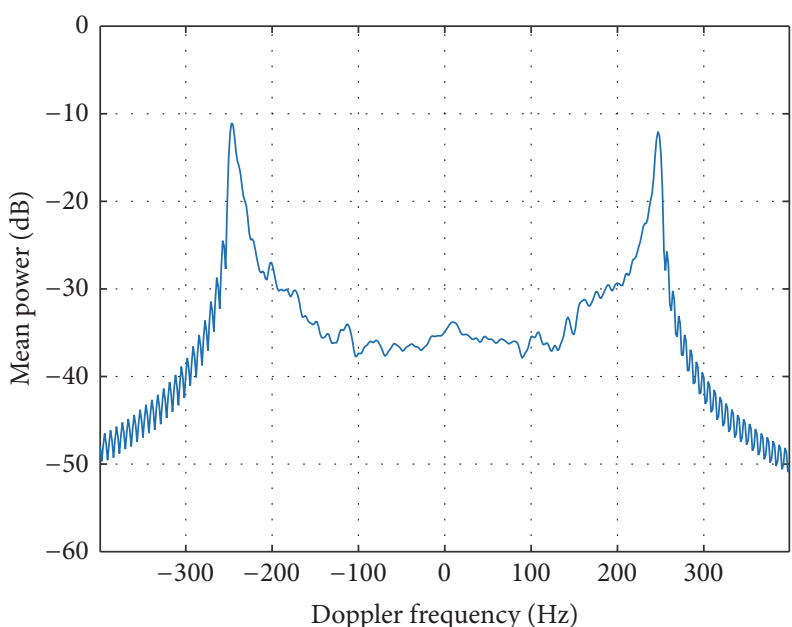

(a) Estimated Doppler PSD for the train moving at $100 \mathrm{~km} / \mathrm{h}$ along segment (a)

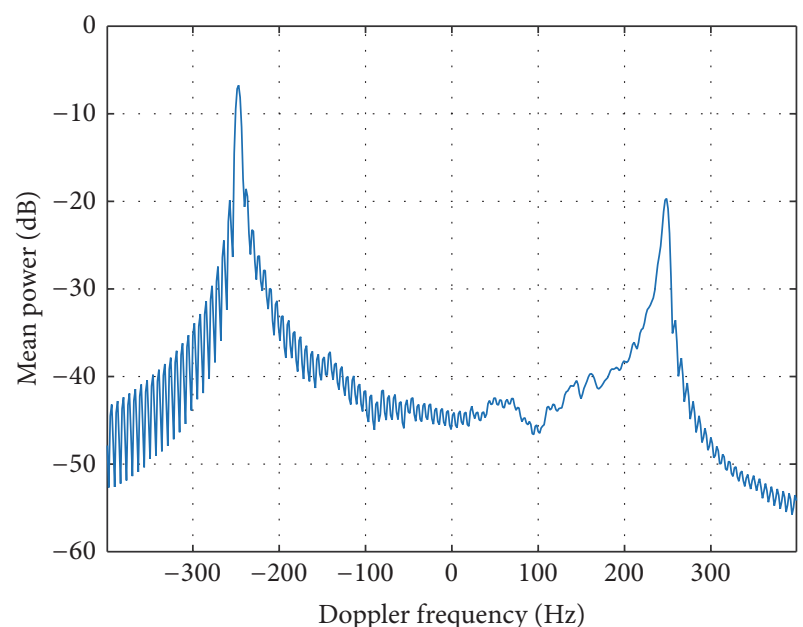

(b) Estimated Doppler PSD for the train moving at $100 \mathrm{~km} / \mathrm{h}$ along segment (b)

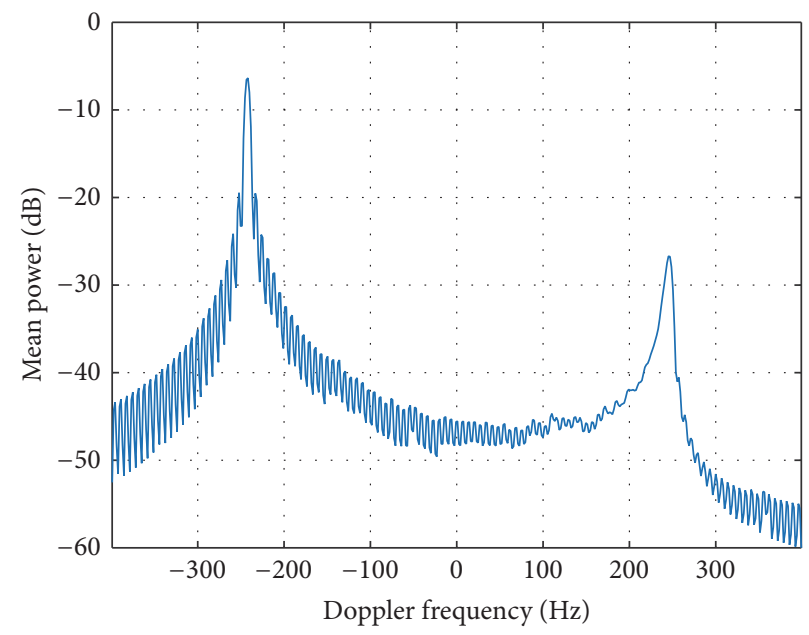

(c) Estimated Doppler PSD for the train moving at $100 \mathrm{~km} / \mathrm{h}$ along segment (c)

FIgURE 24: Estimated Doppler PSD for outdoor antenna 1 for the train moving at $100 \mathrm{~km} / \mathrm{h}$ along segments (a), (b), and (c).

The obtained results exhibit a good level of agreement with the expected ones according to the assumed theoretical models. However, the utmost importance of carrying out measurements from actual LTE-compliant transmit signals is reflected in the so-called particularities found in the considered train scenario. In this paper, we devoted an entire section to study such particularities. More specifically, we observed periodic abrupt transitions in the Doppler PSD along the whole measured path. Such transitions-similar to the ones occurring when the train passes by in front of the eNodeB antennas-are mainly caused by the masts supporting the overhead line. Those effects, which are invariant with the train speed, are also observed in the PDP results. It is worth noting that the Doppler PSD results are distributed approximately according to a "U" shape with an additional peak caused by the LoS component. This shows that, although there is a strong LoS for the considered environment, reflections caused by the railway infrastructure elements may lead to noticeable Doppler components in other frequencies, similar to the Jakes spectrum. Overall, all the condensed parameters are affected by the reflections, as their respective trends change significantly in the areas where the reflections are stronger. Due to this, we can expect severe effective link SNR degradation in such areas.

\section{Conflicts of Interest}

The authors declare that they have no conflicts of interest.

\section{Acknowledgments}

This work has been funded by the Xunta de Galicia (ED431C 2016-045, ED341D R2016/012, and ED431G/01), the Agencia Estatal de Investigación of Spain (TEC2013-47141-C4-1-R, 


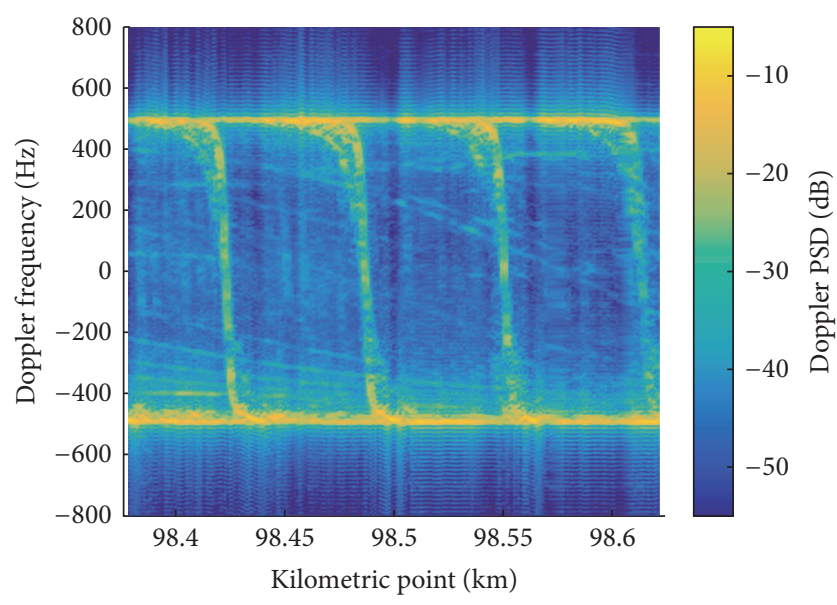

(a) Doppler Spectrum for the train moving at $200 \mathrm{~km} / \mathrm{h}$ along segment (a)

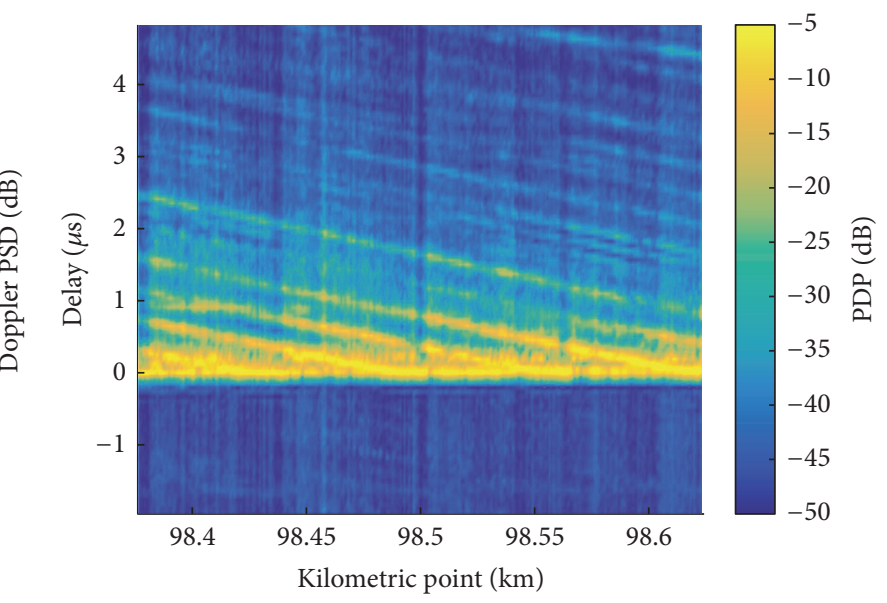

(b) Normalized PDP for the train moving at $200 \mathrm{~km} / \mathrm{h}$ along segment (a). The propagation delay is not taken into account

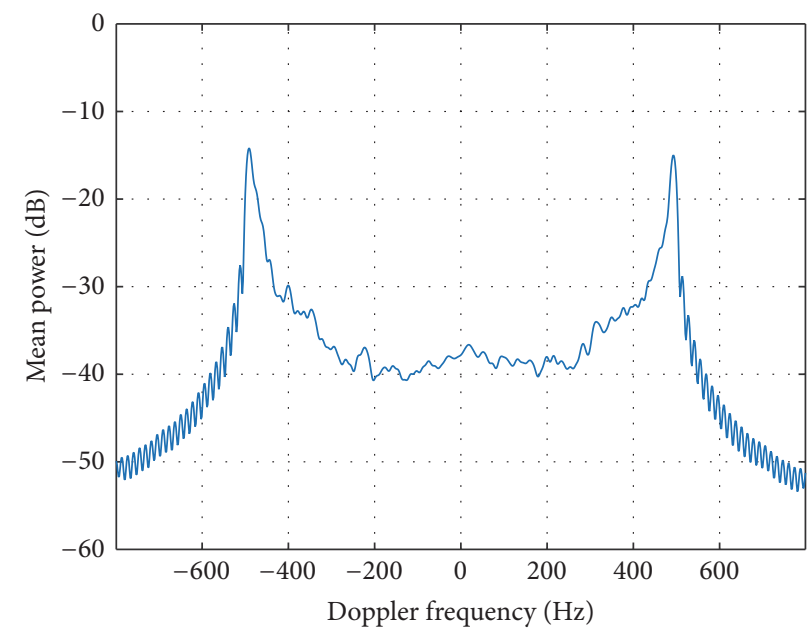

(c) Estimated Doppler PSD for the train moving at $200 \mathrm{~km} / \mathrm{h}$ along segment (a)

Figure 25: Condensed parameters for outdoor antenna 1 for the train moving at $200 \mathrm{~km} / \mathrm{h}$ at segment (a).

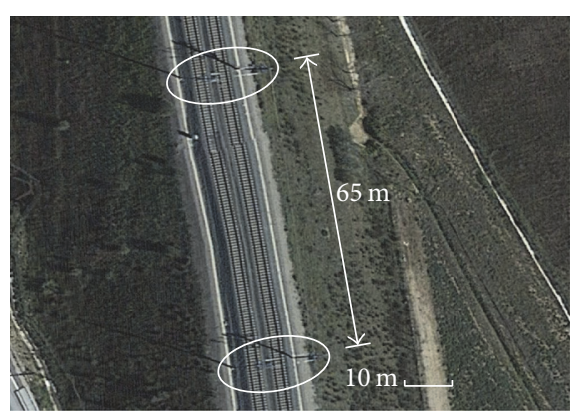

(a) Top view of a small section of the track. The masts supporting the overhead lines are shown enclosed in ellipses. The distance between masts is also shown. Map Image by $\odot 2017$ DigitalGlobe, Cartography Institute of Andalucía, Map Data by (C)2017 Google, National Geographic Institute of Spain

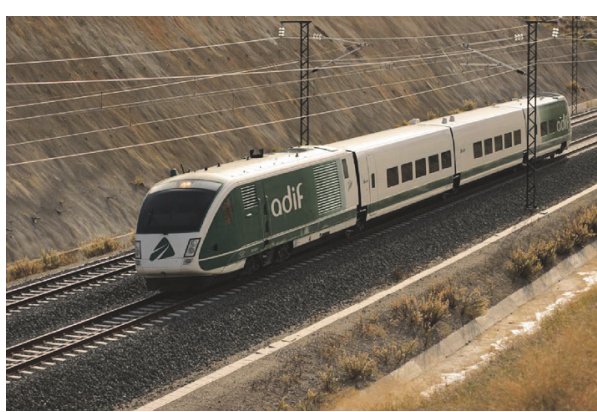

(b) Image of a train on the Córdoba-Málaga line. Some of the masts supporting the overhead line can be seen at both sides of the track. Image obtained from http://www.ferropedia.es licensed under CC BY-SA 3.0

FIGURE 26: Detailed view of the train infrastructure elements that cause signal reflections to the receiver. 
TEC2015-69648-REDC, and TEC2016-75067-C4-1-R), ERDF funds of the EU (AEI/FEDER, UE), and the predoctoral Grant BES-2014-069772. The authors would like to express their gratitude to the personnel from the TECRAIL consortium involved in the measurement campaign.

\section{References}

[1] J. Rodríguez-Piñeiro, Broadband wireless communication systems for high mobility scenarios [Ph.D. thesis], Universidade da Coruña, A Coruña, Spain, 2016.

[2] P. F. Lamas, J. Rodríguez-Piñeiro, J. A. García-Naya, and L. Castedo, "A survey on LTE networks for railway services," in Proceedings of the IEEE Congreso de Ingeniería en ElectroElectrónica, Comunicaciones y Computación (ARANDUCON 2012), Asunción, Paraguay, November 2012.

[3] P. Fraga-Lamas, J. Rodríguez-Piñeiro, J. A. García-Naya, and L. Castedo, "Unleashing the potential of LTE for next generation railway communications," in Proceedings of the 8th International Workshop on Communication Technologies for Vehicles (Nets4Cars/Nets4Trains/Nets4Aircraft 2015), pp. 153164, Sousse, Tunisia, May 2015.

[4] J. Rodríguez-Piñeiro, P. F. Lamas, J. A. García-Naya, and L. Castedo, "TE security analysis for railway communications," in Proceedings of the IEEE Congreso de Ingeniería en ElectroElectrónica, Comunicaciones y Computación (ARANDUCON 2012), November 2012.

[5] "ETCS/GSM-R Quality of Service - operational analysis," 2010, eRTMS Std., 04E117.

[6] "Technical specification group radio access network; mobile relay for E-UTRA," Tech. Rep. 3GPP TR 36.836, 2012.

[7] B. Ai, X. Cheng, T. Kürner et al., "Challenges toward wireless communications for high-speed railway," IEEE Transactions on Intelligent Transportation Systems, vol. 15, no. 5, pp. 2143-2158, 2014.

[8] R. He, A. F. Molisch, Z. Zhong et al., "Measurement based channel modeling with directional antennas for high-speed railways," in Proceedings of the IEEE Wireless Communications and Networking Conference, WCNC 2013, pp. 2932-2936, IEEE, Shanghai, China, April 2013.

[9] R. He, Z. Zhong, B. Ai, and J. Ding, "An empirical path loss model and fading analysis for high-speed railway viaduct scenarios," IEEE Antennas and Wireless Propagation Letters, vol. 10, pp. 808-812, 2011.

[10] R. He, Z. Zhong, B. Ai, L. Xiong, and H. Wei, "A novel path loss model for high-speed railway viaduct scenarios," in Proceedings of the 7th International Conference on Wireless Communications, Networking and Mobile Computing, WiCOM 2011, pp. 1-4, IEEE, Wuhan, China, September 2011.

[11] R. He, Z. Zhong, B. Ai, G. Wang, J. Ding, and A. F. Molisch, "Measurements and analysis of propagation channels in highspeed railway viaducts," IEEE Transactions on Wireless Communications, vol. 12, no. 2, pp. 794-805, 2013.

[12] R. He, Z. Zhong, B. Ai, J. Ding, Y. Yang, and A. F. Molisch, "Short-term fading behavior in high-speed railway cutting scenario: Measurements, analysis, and statistical models," IEEE Transactions on Antennas and Propagation, vol. 61, no. 4, pp. 2209-2222, 2013.

[13] L. Liu, C. Tao, J. Qiu et al., "Position-based modeling for wireless channel on high-speed railway under a viaduct at $2.35 \mathrm{GHz}$," IEEE Journal on Selected Areas in Communications, vol. 30, no. 4, pp. 834-845, 2012.
[14] H. Wei, Z. Zhong, L. Xiong, B. Ai, and R. He, "Study on the shadow fading characteristic in viaduct scenario of the high-speed railway," in Proceedings of the 2011 6th International ICST Conference on Communications and Networking in China, CHINACOM 2011, pp. 1216-1220, IEEE, Harbin, China, August 2011.

[15] T. Zhou, C. Tao, L. Liu, and Z. Tan, "A semi-empirical MIMO channel model for high-speed railway viaduct scenarios," in Proceedings of the 2014 1st IEEE International Conference on Communications, ICC 2014, pp. 5854-5858, IEEE, Sydney, NSW, Australia, June 2014.

[16] Y.Zhang, Z. He, W. Zhang, L. Xiao, and S. Zhou, "Measurementbased delay and doppler characterizations for high-speed railway hilly scenario," International Journal of Antennas and Propagation, vol. 2014, Article ID 875345, 8 pages, 2014.

[17] X. Yin, X. Cai, X. Cheng, J. Chen, and M. Tian, "Empirical geometry-based random-cluster model for high-speed-train channels in UMTS networks," IEEE Transactions on Intelligent Transportation Systems, vol. 16, no. 5, pp. 2850-2861, 2015.

[18] ETSI, ETSI TS 136213 V13.0.0: LTE; E-UTRA; Physical layer procedures, May 2015.

[19] J. Rodríguez-Piñeiro, J. A. García-Naya, A. Carro-Lagoa, and L. Castedo, "A testbed for evaluating LTE in high-speed trains," in Proceedings of the 16th Euromicro Conference on Digital System Design, DSD 2013, pp. 175-182, IEEE, Los Alamitos, CA, USA, September 2013.

[20] T. Domínguez-Bolaño, J. Rodríguez-Piñeiro, J. A. Garcia-Naya, and L. Castedo, "The GTEC 5G link-level simulator," in Proceedings of the 1st International Workshop on Link- and System Level Simulations, (IWSLS2 2016), pp. 64-69, Vienna, Austria, July 2016.

[21] "GTEC Testbed Project," https://bitbucket.org/tomas_bolano/ gtec_testbed_public.git, 2017.

[22] ETSI, ETSI TS 136211 V14.2.0: LTE; E-UTRA; Physical Channel and Modulation, April 2017.

[23] S. Sesia, I. Toufik, and M. Baker, LTE The UMTS Long Term Evolution from Theory to Practice, John Wiley \& Sons, 2nd edition, 2011.

[24] P. J. Huber, Robust Statistics, Wiley-Interscience, 1981.

[25] T. S. Rappaport et al., Wireless Communications: Principles and Practice, vol. 2, Prentice Hall PTR, New Jersey, USA, 1996.

[26] L. J. Greenstein, D. G. Michelson, and V. Erceg, "Momentmethod estimation of the Ricean $K$-factor," IEEE Communications Letters, vol. 3, no. 6, pp. 175-176, 1999.

[27] A. F. Molisch, Wireless Communications, vol. 34, John Wiley \& Sons, 2012.

[28] J. WC Jr., “Microwave mobile communications," 1974. 


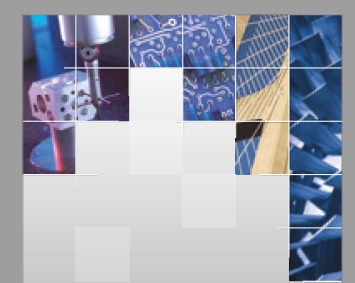

\section{Enfincering}
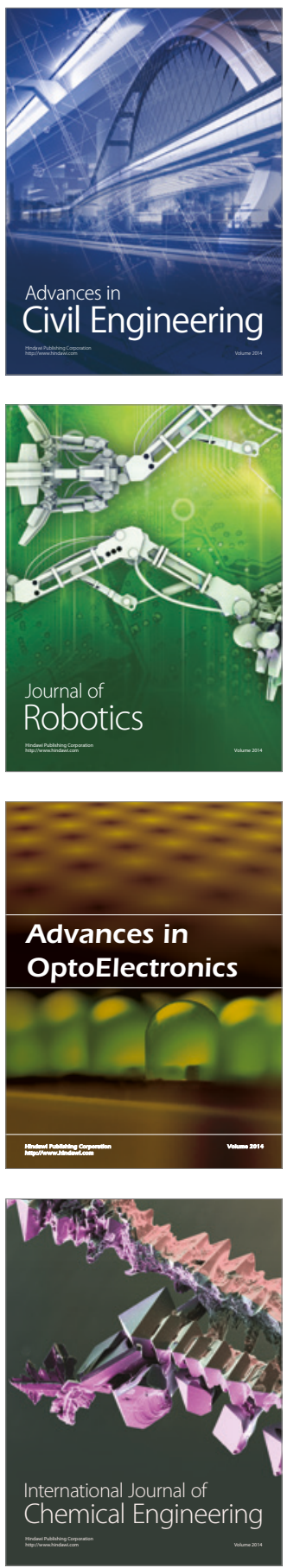

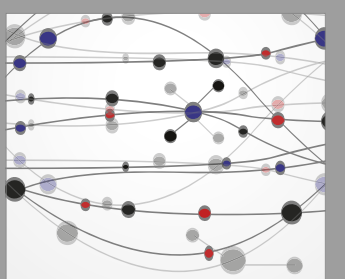

The Scientific World Journal

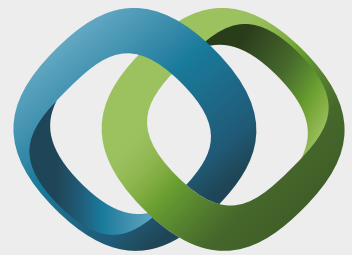

\section{Hindawi}

Submit your manuscripts at

https://www.hindawi.com
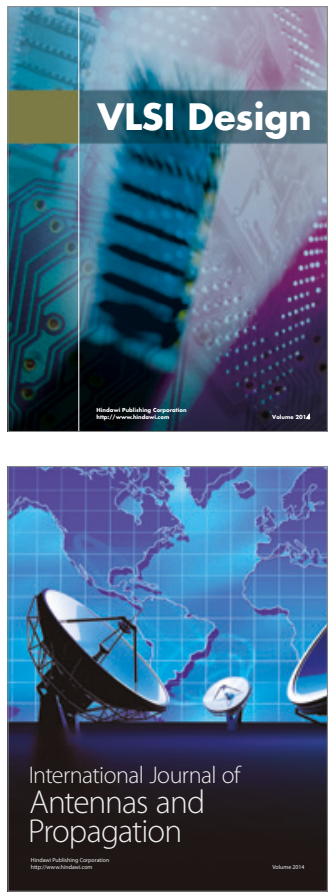

\section{Rotating}

Machinery
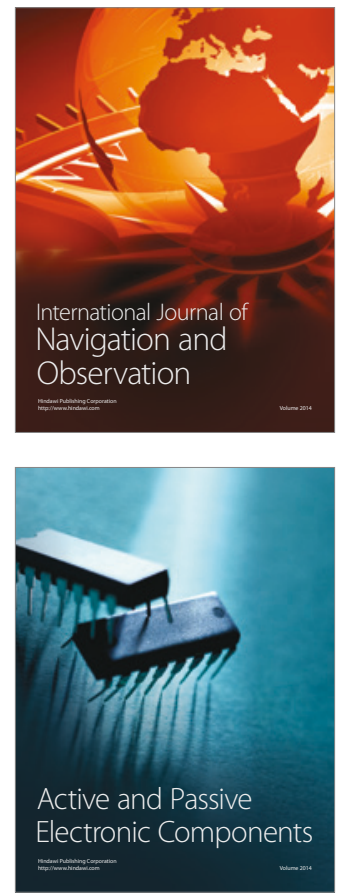
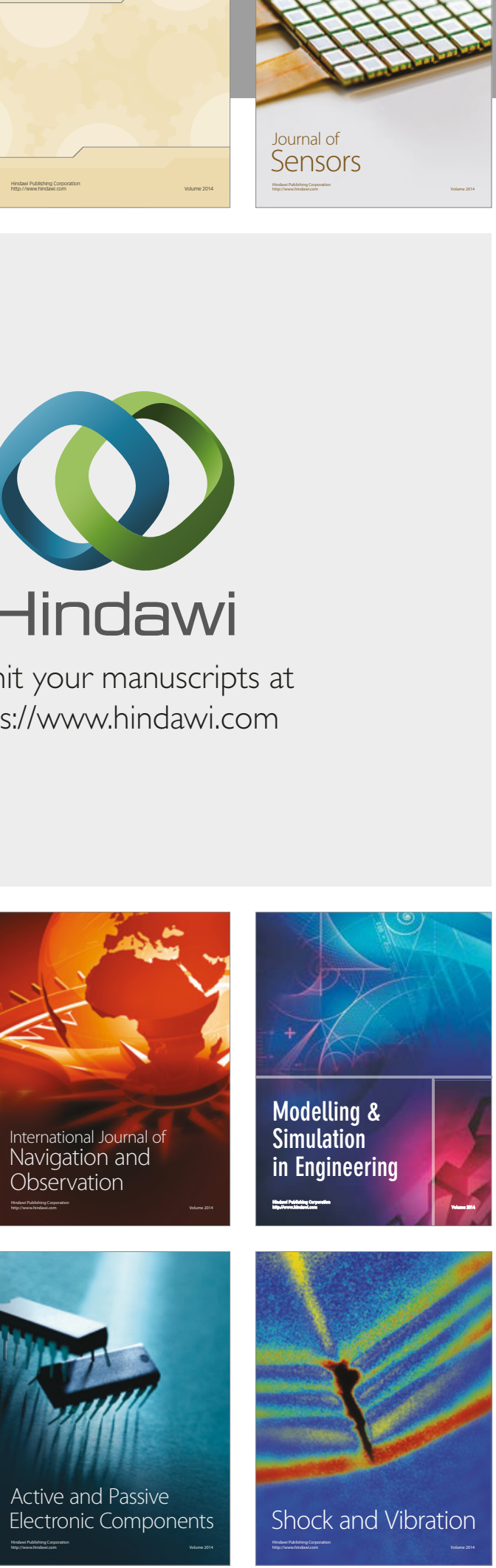
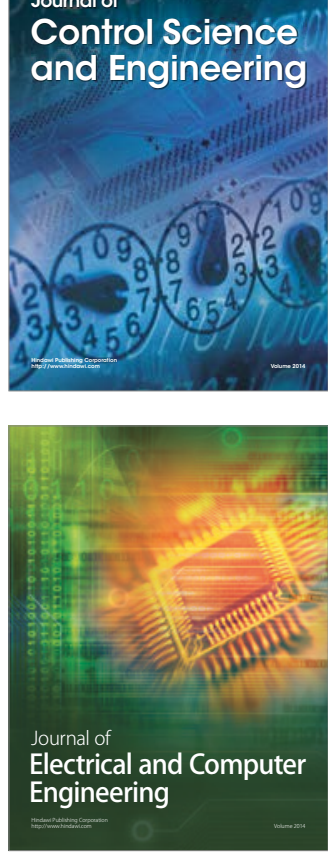

Distributed

Journal of

Control Science

and Engineering
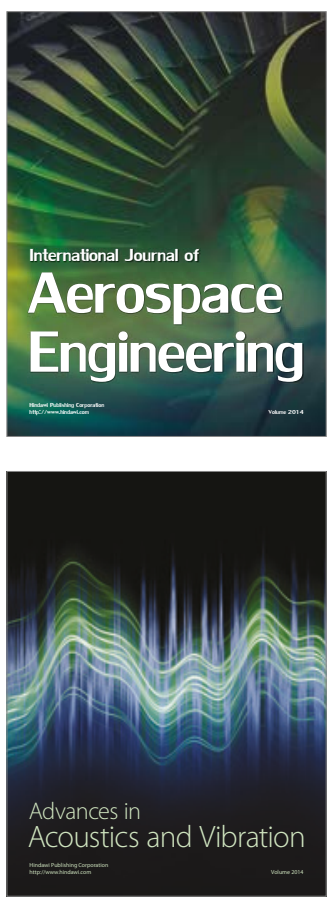

Sensor Networks 